\title{
Reflection Of Microwave Pulses From Acoustic Waves: Summary of Experimental and Computational Studies
}

\author{
G.M. Kepler* ${ }^{*}$, R.A. Albanese ${ }^{\S}$, H.T. Banks ${ }^{\ddagger}$, and V.A. Bokil ${ }^{\dagger}$ \\ 『 Center for Research in Scientific Computation, \\ North Carolina State University, \\ Raleigh, N.C. 27695-8205, USA \\ "Human Effectiveness Directorate, \\ Biomechanisms and Modeling Branch, \\ AFRL, Brooks AFB, TX 78235, USA
}

*email: gmkepler@ncsu.edu

†email: htbanks@ncsu.edu

‡email: vabokil@ncsu.edu

$\S$ email: richard.albanes@he.brooks.af.mil 


\section{INTRODUCTION}

In $[1,4]$, the authors proposed and analyzed an interrogation (inverse problem) methodology based on use of an acoustic wave as a reflecting virtual interface for propagating impulses. It is by now well accepted (e.g., see $[2,7,11,14]$ ) that acoustic pressure waves will interact with electromagnetic signals in ways that often mimic interfacial partial reflection/partial transmission for the electromagnetic waves. The response of atomic electrons to an applied electrical field in a material medium results in a material polarization with a concomitant index of refraction that is a function of the local density in the material. Thus, material density fluctuations produced by a sound wave induce perturbations in the index of refraction. Previous computational work in $[1,3]$ suggested that it might be possible to detect reflections of microwave frequency EM waves from a slowly (relative to the speed of the EM wave) moving acoustic wave front. These efforts focused on reflections in a Debye medium. The authors made an argument for a simple pressure dependent dielectric model in which the Debye parameters exhibit a linear acoustic pressure dependence. In [1], finiteelement simulations for a simple 1D geometry demonstrated computationally that EM reflections from the acoustic pulse are possible. These findings were confirmed with $2 \mathrm{D}$ computations in [3]. The results of $[1,3]$ consisting of a theoretical framework as well as computational validation of such an approach provide ample motivation for significant "proof-of-concept" experimental investigations of the proposed methodology. These prompted our preliminary experiment on which we report here to look for microwave frequency EM reflections from an acoustic pulse.

A successful demonstration of such a technology is important. There are many potential applications for detection of electromagnetic (EM) pulses off acoustic waves, such as nondestructive evaluation of materials, detection of underground bunkers and mines, and medical imaging. One generates the electrical property distributions in the body (Microwave maps) with the hope that such properties of different bodily tissues are related to their physiological state. With such noninvasive interrogating techniques one can study properties and defects in biological tissues with very little discomfort to the subjects. Other potential applications for such interrogation techniques are nondestructive damage detection in structures where very high frequency electromagnetic pulses can be used to detect the location and width of cracks that may be present [5]. Additional applications are found in mine, ordinance and camouflage surveillance, and subsurface and atmospheric environmental modelling.

Presently, ultrasonic acoustic waves are used in medical imaging of the heart, fetus, etc. Similarly, EM waves are employed in the form of CT scans and x-rays. Each method has advantages that make it the method of choice for visualization of particular body structures and conditions. One can then imagine that combining the two techniques and detecting EM reflections from acoustic fronts might also have its own usefulness for particular scenarios.

This report represents a summary of our experimental investigations to detect the scattering of microwave frequency electromagnetic waves by induced acoustic structures. The essence of this experiment consists of launching electromagnetic and acoustic waves that will be coincident in a target and using time domain reflectometry (TDR) measurements to detect reflections of the electromagnetic wave off the acoustic wave front. 


\section{EXPERIMENTAL SET-UP}

The guiding wave structure is a TEM half plane antenna, as shown in Figure 1. An electromagnetic wave is launched at the feed (narrow end) of the antenna by means of a fast rise-time (200 ps) input pulse from an AVTECH AV-1030-C pulse generator. The TEM antenna guides the spherical wave to a target placed near the aperture (large end) of the antenna. There are two components to the antenna design: the expanding and parallel-plate portions. In the expanding portion of the antenna, the height and width of the antenna are increasing so that the expanding spherical wave will more closely approximate a plane wave when it hits the target. The target is placed in the parallel-plate portion of the antenna, which may be terminated with resistive wires connecting the hot plate to the ground plane.

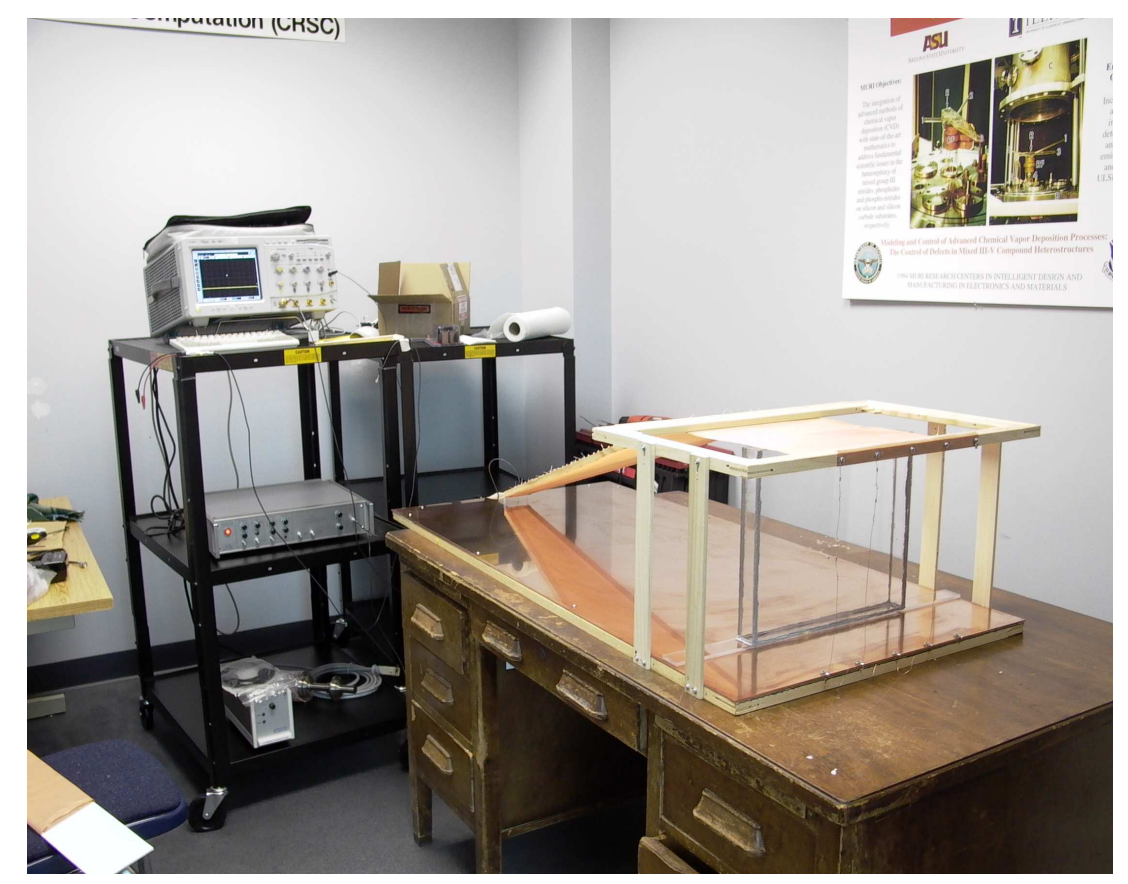

Figure 1: The antenna and some of the related equipment. An empty plexiglass container is placed in the parallel portion of the antenna to illustrate the placement of the agar target. Resistive end termination wires can be seen across the aperture.

Figures 2 and 3 are schematic diagrams of the antenna. The ground plane consists of a $1 / 8$ inch thick electronic grade copper plate supported on a wooden frame, while the hot plate is constructed from 100 mesh copper wire cloth secured to a wooden frame with $30 \mathrm{lb}$. monofilament fishing line. The hot plate is $2.75 \mathrm{in}$. wide at the feed of the antenna and $12 \mathrm{in.}$ wide at the aperture. The spacing between the hot plate and the ground plane is $0.5 \mathrm{in}$. at the feed and $12 \mathrm{in}$. at the aperture.

The agar slab dimensions are $13.00 \times 11.25 \times 1.00$ inches, although some measurements were performed with a thicker (4 in.) agar slab. The outer dimensions of the plexiglass box containing the agar slab are $13.75 \times 12.00 \times 1.75$ inches. A one half inch diameter hole is cut into the center of the back wall (wide end) of the plexiglass container. A hollow plexiglass cylinder ( $\frac{3}{4}$ inch length and outer diameter) is centered on the hole and projects out from the container. The acoustic transducer (ITC-215 kHz air transducer) is inserted into the cylinder and and wedged in to make firm contact with the back of the agar slab. For the measurements reported here, the agar slab was placed in the antenna such that the front of the plexiglass container (facing the feed) was $7.25 \mathrm{in}$. from the aperture (end) of the antenna. Therefore, the front surface of the agar was approximately 44.125 in. from the feed of the antenna. 


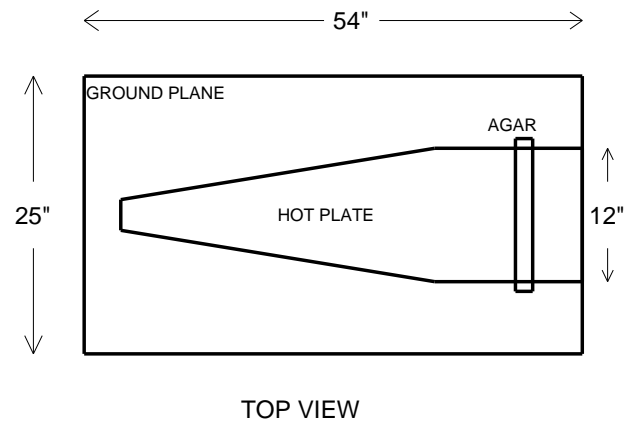

Figure 2: Top view of antenna with agar slab (not to scale).

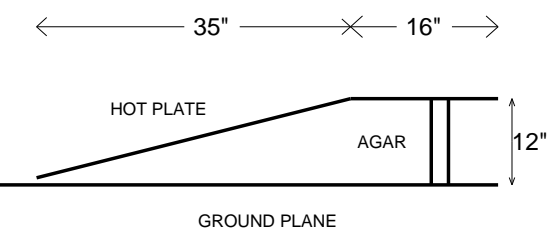

SIDE VIEW

Figure 3: Side view of antenna with agar slab (not to scale).

A block diagram of the experimental setup is shown in Figure 4. Time domain reflectometry (TDR) measurements are used to measure the reflections of the electromagnetic pulse from structures inside the antenna.

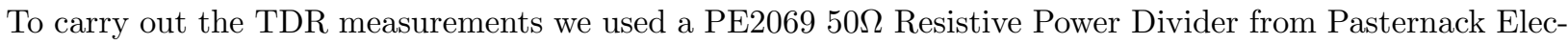
tronics. The power divider has a frequency range of DC-6 GHz, an insertion loss of $7 \mathrm{db}$, and a max VSWR of 1.20:1. The $50 \Omega$ power divider splits the signal with minimal reflections. The input pulse from the Signal Generator travels through an RG-174 cable to the underside of the ground plane, where it connects to an SMA Female Bulkhead connector (PE4101 Pasternack) mounted through the ground plane. The inner conductor of the connector feeds through the ground plane and is connected to the hot plate by means of a copper wire connection. Referring to the experimental set-up in Figure 4, it can be seen that we measure

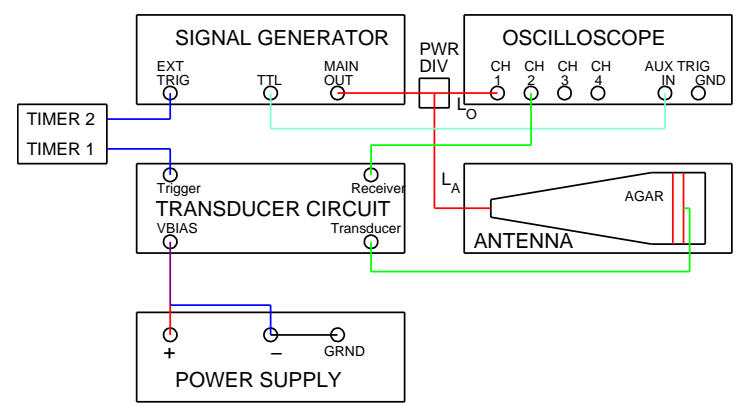

Figure 4: Diagram of the TDR experimental set-up. $L_{O}=6 \mathrm{ft}$ and $L_{A}=6$ or $12 \mathrm{ft}$.

the input pulse after the signal is divided and, subsequently, measure reflected signals coming back from the antenna. These measurements are accomplished using an Agilent Infinium 54854A oscilloscope that is triggered by a TTL signal from the Signal Generator. 


\subsection{Frequency Composition of Input Pulse}

A typical microwave input pulse to the antenna is plotted in Figure 5. The input pulse is approximately $8 \mathrm{~ns}$ wide and has a peak voltage of $2 \mathrm{~V}$. Information about the underlying frequency composition of the input pulse is obtained using a fast-fourier transform (FFT) of the input pulse (Fig. 6). Plotted on a log-log scale, it can be seen that the input power drops linearly as a function of frequency in the range $40 \mathrm{MHz}-$ $4 \mathrm{GHz}$. Above $4 \mathrm{GHz}$ there is a steep drop-off in power. Interestingly, there seems to be a small burst of power around $20 \mathrm{GHz}$.

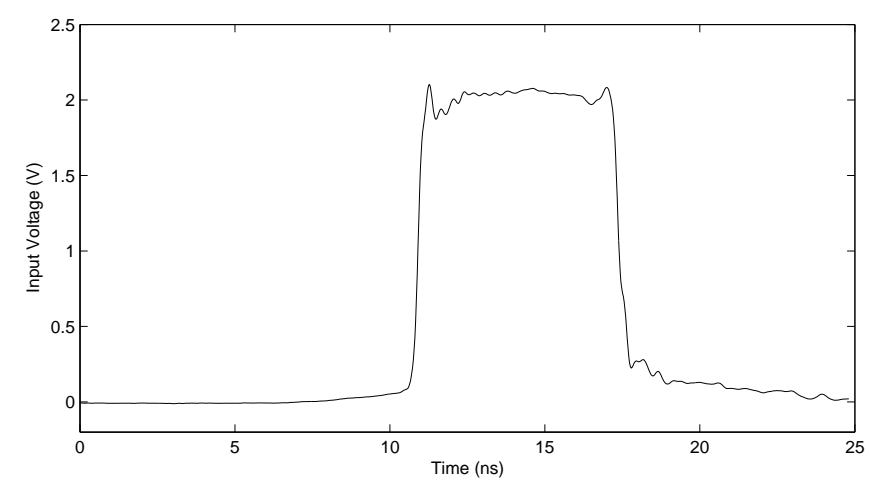

Figure 5: A typical input pulse.

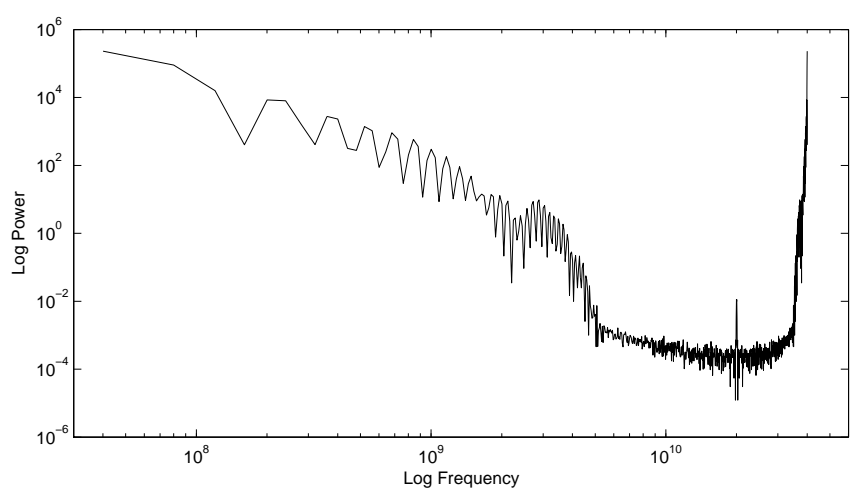

Figure 6: FFT of the input pulse above.

\section{EMPTY ANTENNA RESULTS}

We first investigated reflections from structures in the antenna in the absence of an acoustic target using TDR measurements. Figure 7 compares data for the two cases of $L_{A}=6$ and $12 \mathrm{ft}$ (see Fig. 4). The initial pulses $(t=0)$ for the $L_{A}=6$ and $12 \mathrm{ft}$ cases coincide, as expected, representing the portion of the signal generator pulse that travels directly from the splitter to the oscilloscope. The (time) positions of subsequent pulses depends upon the coaxial cable length $L_{A}$.

The first reflected pulse occurs at times $t_{1}=19$ and $38 \mathrm{~ns}$ for the $6 \mathrm{ft}$ and $12 \mathrm{ft}$ cables, respectively. This pulse occurs because of reflections at the coaxial cable/antenna connection and indicate imperfect impedance matching between the feed and the $50 \Omega$ coaxial cable. The pulse reflected at the coax/antenna junction has the same orientation as the incident pulse. The reflection coefficient is 0.186 (incident voltage/reflected voltage). 
The second reflected pulse corresponds to reflections of the transmitted pulse from the end of the antenna. Reflections from the open-ended antenna maintain the same orientation. After the pulse reflects from the end of the antenna a portion is transmitted through the feed to the oscilloscope (incident time $t_{2}=t_{1}+t_{A}$ ), while the rest is re-radiated in the antenna, but with an inverted portion. Subsequent reflected pulses alternate in orientation, each occurs at a time $t_{A}$ after the next.

The time of arrival of the reflected pulses can be calculated using information about the speed of propagation in the RG174 coaxial cables $\left(1.9 \times 10^{8} \mathrm{~m} / \mathrm{s}\right)$ and in air $\left(3.0 \times 10^{8} \mathrm{~m} / \mathrm{s}\right)$ and the dimensions of the antenna. Table 2 tabulates calculated values for time of arrival of reflection pulses, where it can be seen that the calculated values are in good agreement with measurements (Fig. 7).

052604 data. PE2069 50 ohm Tee data. 1 ave. No agar. No coax sensors.
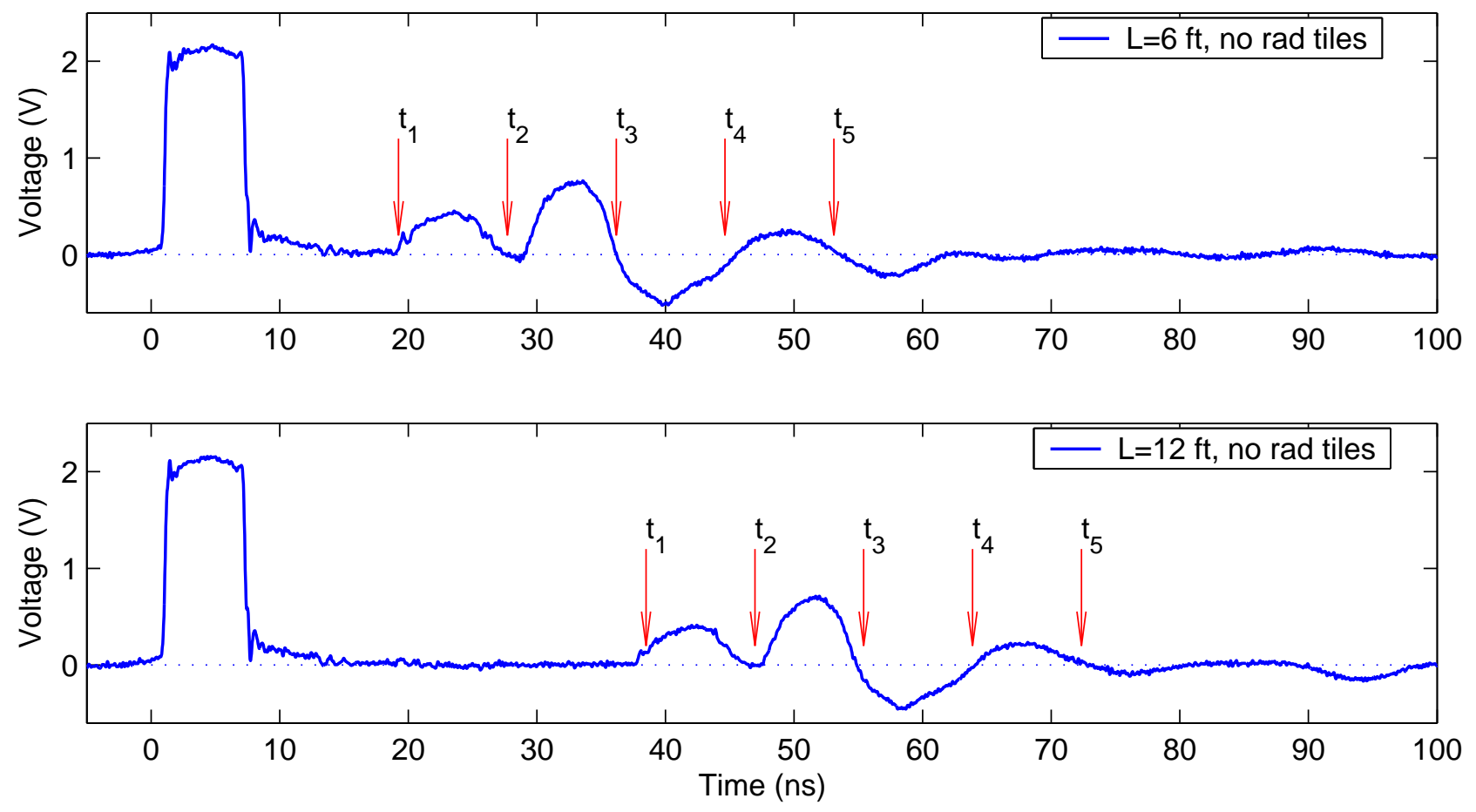

Figure 7: TDR experiment for the two cases of a $6 \mathrm{ft}$ and $12 \mathrm{ft}$ cable from the power divider to the antenna. Calculated reflection times are indicated by the arrows.

Table 2.Round-trip Times

\begin{tabular}{lcc} 
& $\begin{array}{c}\text { PULSE ARRIVAL TIMES } \\
\text { (CALCULATED) } \\
6 \mathrm{ft} \mathrm{COAX} \\
(\mathrm{ns})\end{array}$ & $\begin{array}{c}12 \mathrm{ft} \mathrm{COAX} \\
(\mathrm{ns})\end{array}$ \\
\hline$t_{1}=t_{C O A X}$ & 19.25 & 38.5 \\
$t_{2}=t_{C O A X}+t_{A}$ & 27.71 & 46.96 \\
$t_{3}=t_{C O A X}+2 t_{A}$ & 36.17 & 55.42 \\
$t_{4}=t_{C O A X}+3 t_{A}$ & 44.63 & 63.88 \\
$t_{5}=t_{C O A X}+4 t_{A}$ & 53.09 & 72.34
\end{tabular}




\subsection{Radiation Absorbing Tiles}

Figure 8 compares the reflected pulses with and without radiation absorbers (Braden Shielding Systems) at the aperture of the antenna. No significant difference can be seen between the two cases. The combination of ferrite tiles and pyramidal ferrite absorbers that was used provides a broad range of power absorption in the frequency range of the antenna. Therefore, these results suggest that the radiated portion of the electromagnetic pulse, which is being absorbed by the tiles, is largely independent of the reflected signal in the TDR measurements. Despite having little affect on the reflected signal, the radiation tiles were placed at the aperture of the antenna for all the EM/acoustic interaction data reported here.

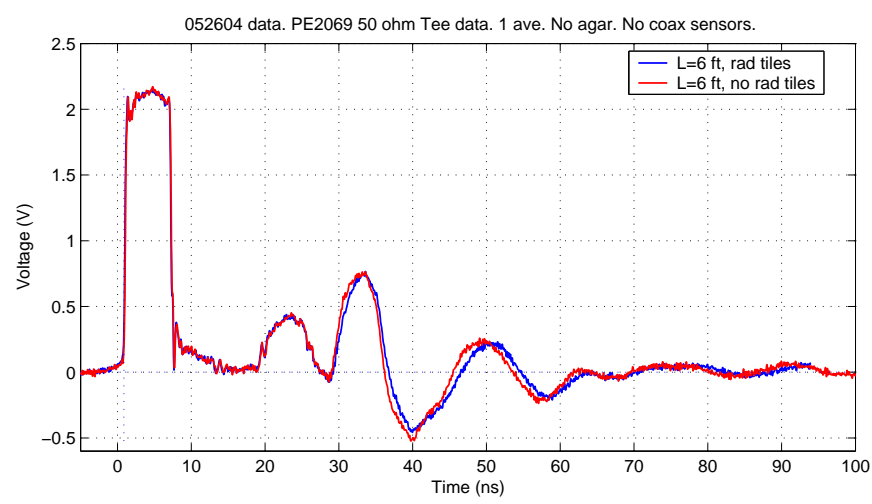

Figure 8: TDR measurements with (blue) and without (red) radiation absorbing tiles at the aperture.

\subsection{Resistive End Termination}

In the next set of experiments we seek to reduce the reflections inside the antenna by varying the end termination at the aperture end of the antenna. Four or five resistors (5\% tolerance, $1 / 4 \mathrm{Watt})$ are connected in parallel from the hot plate to the ground plane. Figure 9 plots the TDR results for different values of (total) resistance between the hot plate and the ground plane. The first peak in Fig. 9(a) is the (split) pulse directly from the Signal Generator. The second peak is the reflection of the other half of the split pulse at the coaxial cable/antenna feed interface. As expected, this peak is independent of the end termination. The third peak/valley and subsequent features represent repeated reflections of the pulse from the end of the antenna. These features will be greatly affected by the end termination, since reflections from the end of the antenna will be minimized when the end termination resistance matches the characteristic impedance of the antenna at the aperture. From Fig. 9 it is evident that the end reflections are minimized with an end termination of approximately $125 \Omega$.

These results suggest that the characteristic impedance of the antenna at the aperture end is approximately $125 \Omega$. The antenna was designed to have a $377 \Omega$ characteristic impedance at the aperture in order to be impedance matched to the characteristic impedance of radiation in free space. This design was based on the assumption that the characteristic impedance of the end configuration most closely matched that of a parallel plate configuration (Fig. 10a). Because the ground plane extends out beyond the hot plate, our configuration may more closely resemble that of a microstrip (Fig. 10b). Using the microstrip formula, the characteristic impedance of the antenna at the aperture end ( $H=12$ and $W=12$ inches) is expected to be $Z_{0}=125 \Omega$, which agrees well with the end termination resistance results (above). 

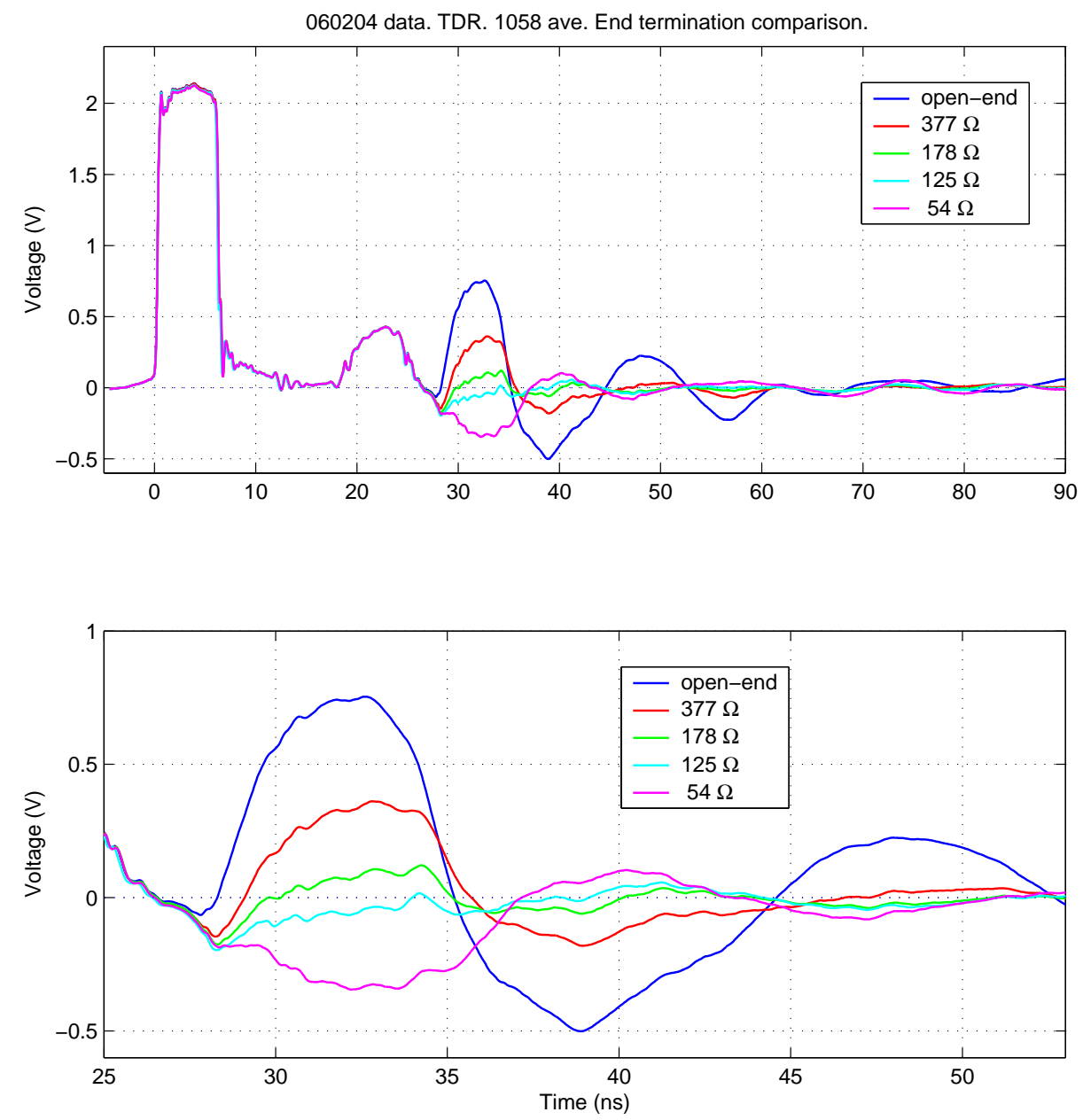

Figure 9: TDR results with different resistive end-terminations at the antenna aperture.

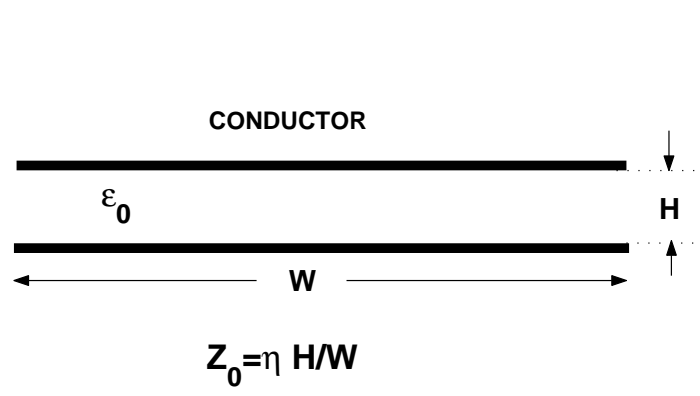

(a) Parallel Plate.

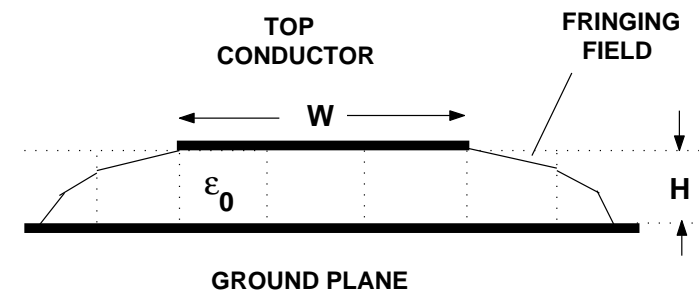

$\mathrm{Z}_{0}=\eta /(\mathrm{W} / \mathrm{H}+2)$

(b) Microstrip.

Figure 10: Two different configurations that approximate the characteristic impedance of the antenna at the aperture end The dimensions of the antenna are $H=12$ and $W=12$ inches at the aperture. In the equations $\eta=\sqrt{\mu_{0} / \epsilon_{0}}=377 \Omega$ is the characteristic impedance of free space. 


\section{ACOUSTIC WAVES}

\subsection{Transducer Characterization}

The ultrasonic transducer also acts as a receiver of acoustic waves. The receiver capability can be used to check that the transducer is functioning properly and to measure the velocity of sound in the agar target. Figure 11 plots the signal that is received by the transducer as a function of time for different target distances $(D)$. The initial portion of the signal is the same for all cases and represents the ring-down of the pulse in the transducer. The secondary pulses represent the reflection of the acoustic signal from a hard target. As expected, the locations (round-trip times) of the reflected pulses increase as the target distance increases.

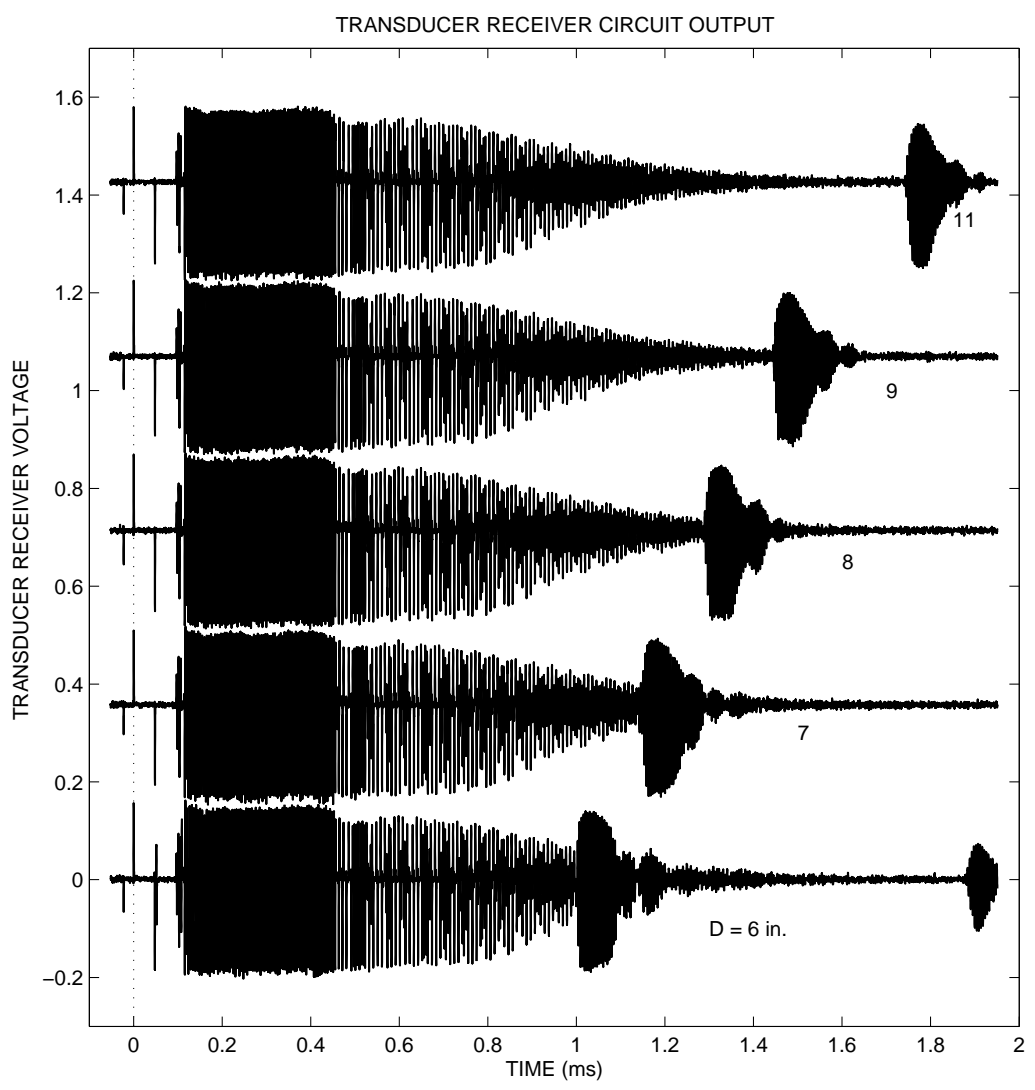

Figure 11: Output of the received transducer signal (Volts) as a function of time for different target distances $(D)$.

The time of arrival of the reflected pulse is plotted as a function of the target distance in Figure 12 (circles) along with the calculated round-trip time (line). Offsetting the calculated times by $0.11 \mathrm{~ms}$ gives good agreement between the observed and calculated arrival times. The offset time probably represents a fixed delay time in the circuit. 


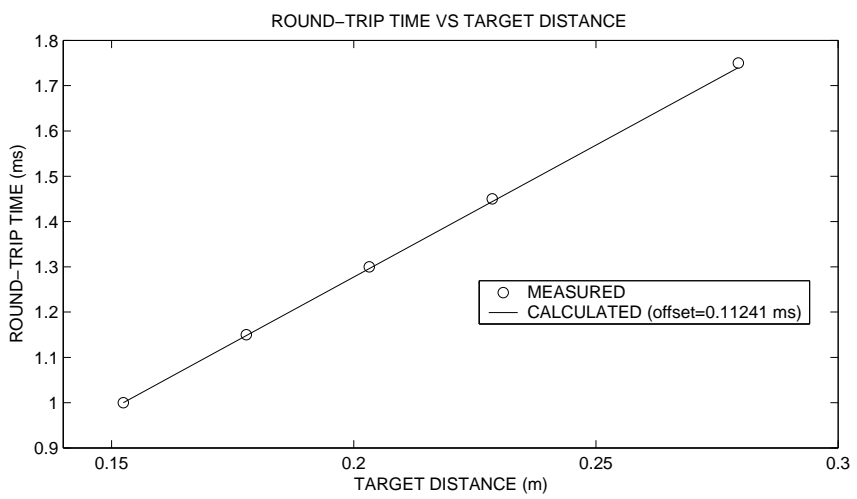

Figure 12: Observed (circles) and calculated (line) arrival times for the reflected acoustic pulses as a function of the target distance $D$.

\subsection{The Velocity of Acoustic Waves in Agar}

To measure the velocity of the acoustic waves in agar we mount the transducer on the top (narrow) surface of agar and point it down into agar. The acoustic pulse travels $0.29 \mathrm{~m}$ to the bottom plexiglass surface, where it is reflected back to the transducer. The reflected signal received by the transducer is shown in Figure 13 (black curve). The initial portion of the signal (0-0.5 ms) represents the ring-down period of the transducer receiver circuit. There are many oscillations in the signal from the receiver circuit, but distinct reflection "pulses" are evident. It is easier to look at a min/max smoothed plot of the data shown above.

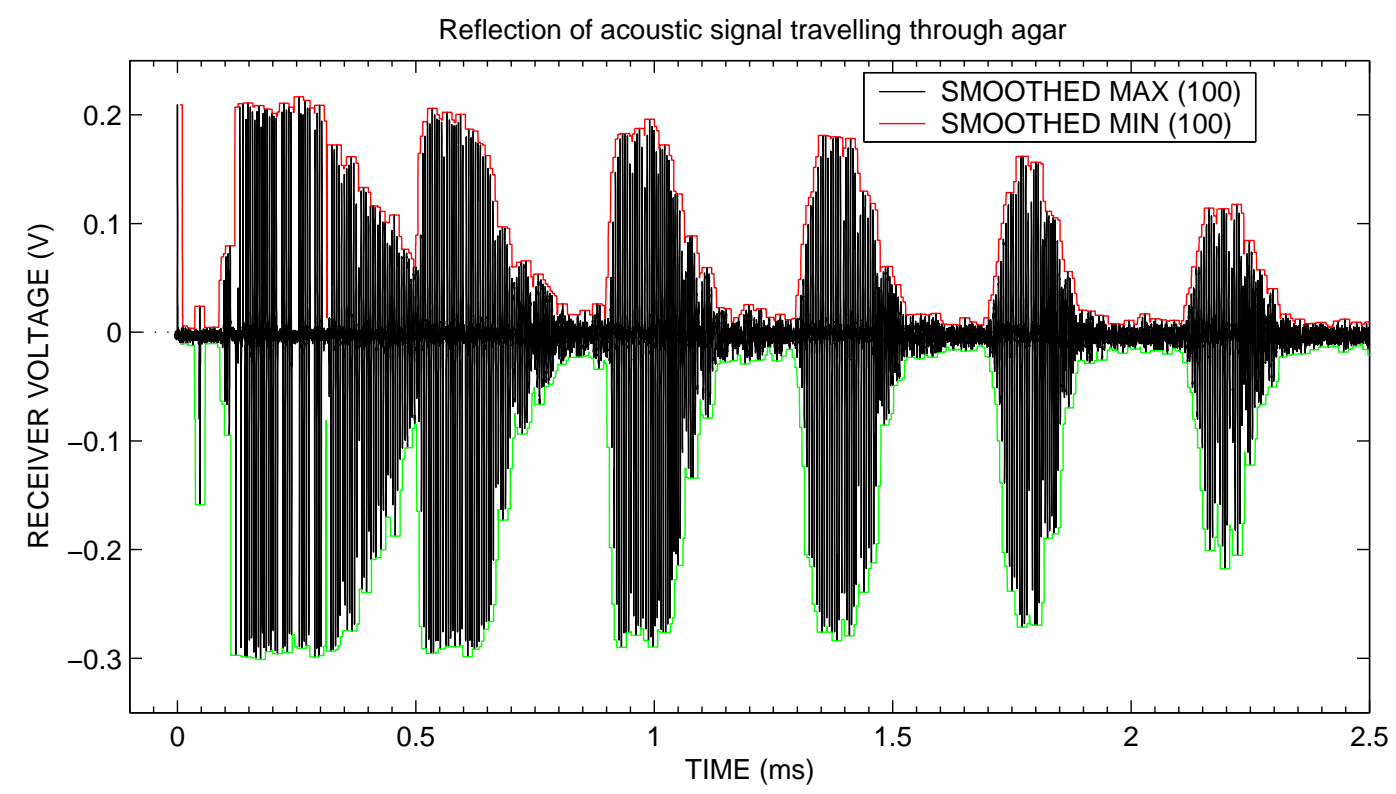

Figure 13: Oscilloscope measurement of transducer receiver circuit signal for the case of an acoustic wave travelling through agar. Reflections from the opposing plexiglass surface can be seen at $0.5 \mathrm{~ms}$ and at approximately $0.4 \mathrm{~ms}$ intervals thereafter. The red and green curves show the min/max smoothed curved for this data $(N=100)$.

At each time point the plotted value equals the maximum (red curve) or minimum (green curve) value of the current point and $N$ points ahead and behind the current point (Figure 14 with $N=100$ ). The velocity of the acoustic wave in agar is calculated to be the round-trip distance in agar divided by the time between reflections. Here the velocity is measured to be $1450 \mathrm{~m} / \mathrm{s}$, which is consistent with published values. 


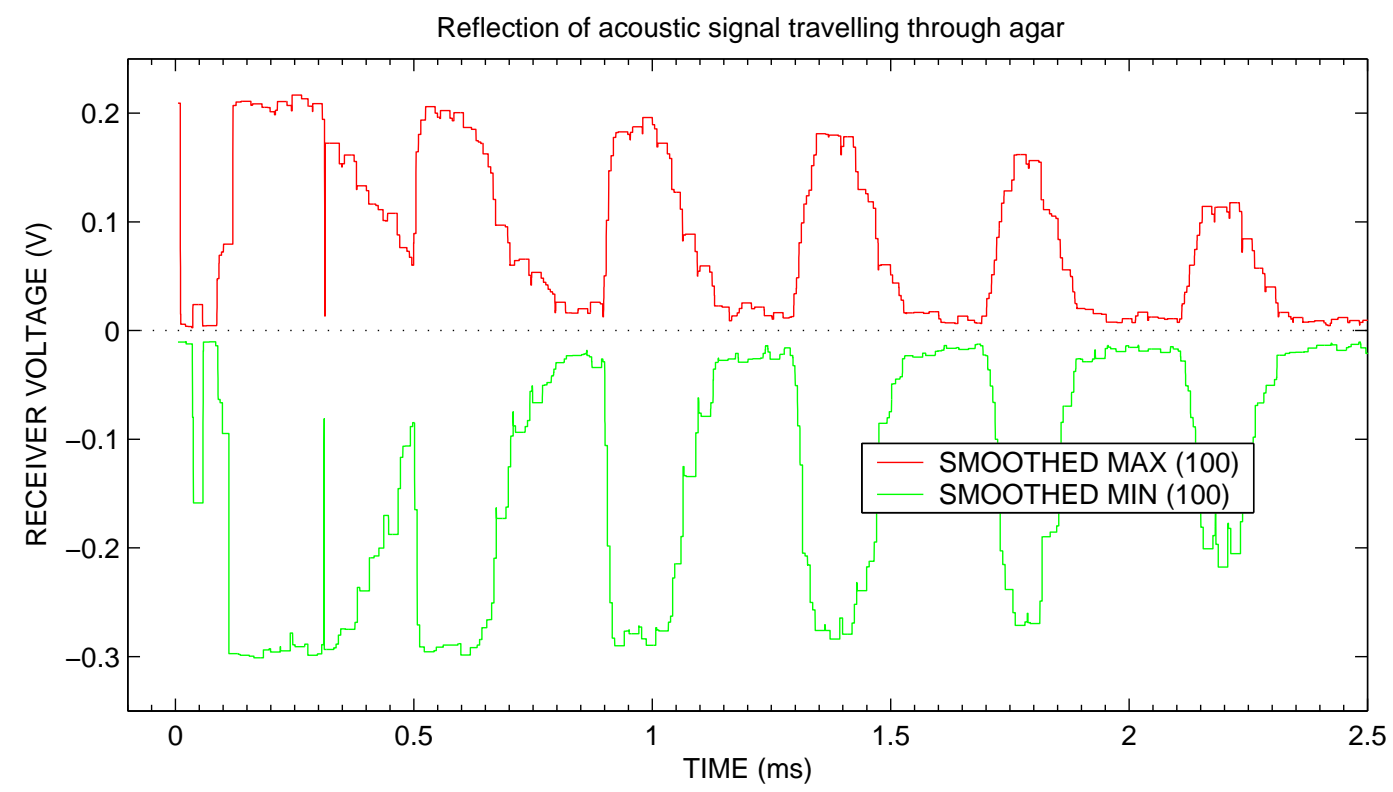

Figure 14: The min/max smoothed plot of the signal from the transducer receiver circuit showing reflections of the acoustic pulse from the plexiglass surface.

\subsection{Comparison of the Transducer Receiver Signal in Agar and Air}

The transducer is mounted against the back of the agar as is the case when measuring EM/Acoustic interactions. The acoustic pulse travels 1 inch before reflecting from the opposing plexiglass surface. The transducer is aimed at a distant point outside the antenna (in air). No reflections are evident in the air, because of the large distance. The reflections in agar are spaced at $50 \mu$ s intervals. The spacing should be $35 \mu \mathrm{s}$.

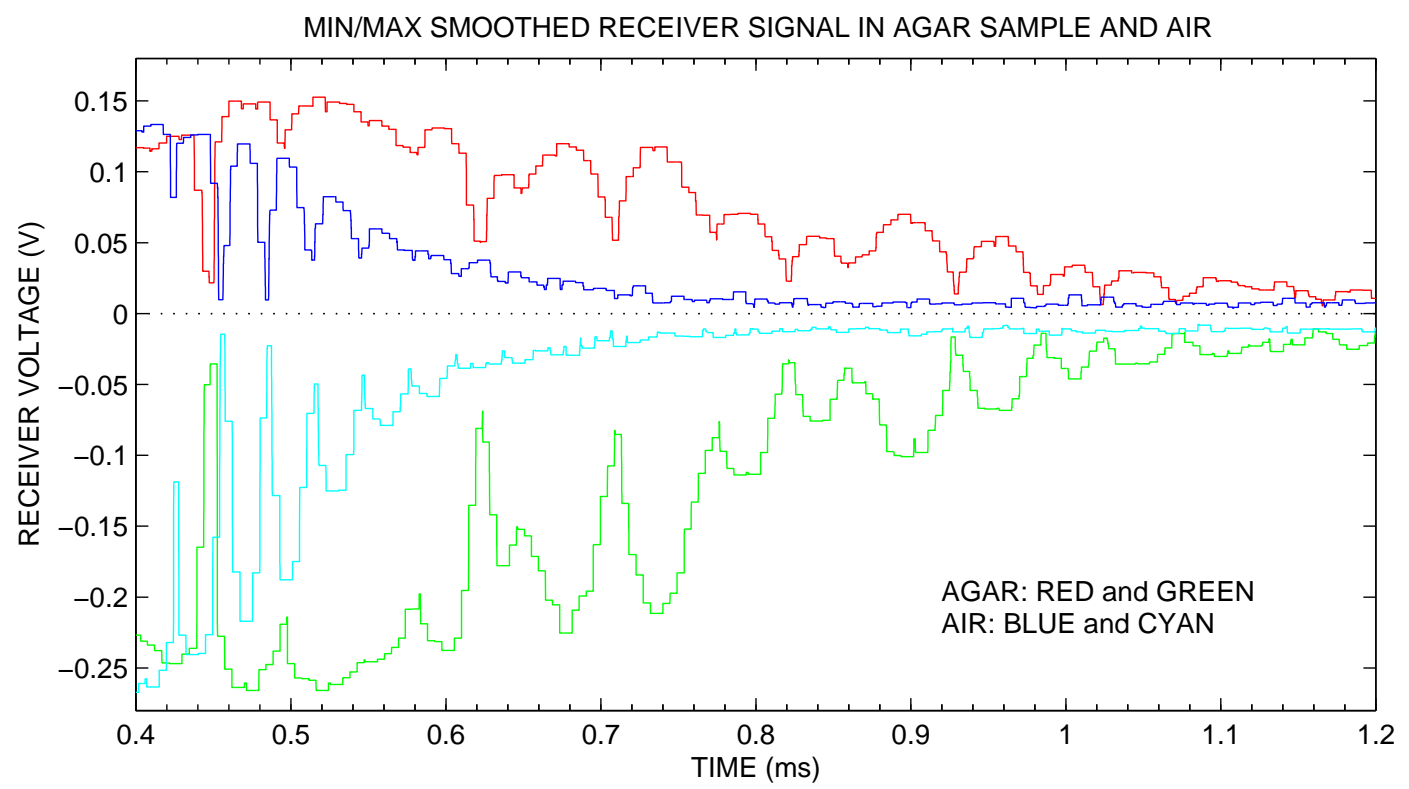

Figure 15: The signal from the transducer receiver circuit for a pulse traveling in air (blue and cyan curves) as compared to a pulse traveling in the agar (red and green curves). 


\section{SYNCHRONIZATION OF EM AND ACOUSTIC WAVES}

In order to have the electromagnetic and acoustic waves coincident in the antenna we must have allow a sufficient delay for launching the electromagnetic wave after the acoustic wave is launched, since the velocity of the electromagnetic wave is much greater than that of the acoustic wave.

The synchronization is accomplished with two timer circuits and a variable delay time control on the Signal Generator (Fig. 4). The first timing circuit triggers the second timing circuit as well as providing the trigger input for the transducer circuit. The second triggering circuit serves as an external trigger to the Signal Generator. Synchronization of the acoustic and EM pulses is achieved by selecting an appropriate value for the delay time on the Pulse Generator. The delay time is the time between the arrival of the External Trigger pulse and the output of the EM pulse from the Pulse Generator (Fig. 16). ${ }^{1}$ Since the speed of the acoustic pulse in the agar is approximately $17 \mu \mathrm{s} /$ inch and the transducer trigger pulse is $134 \mu \mathrm{s}$, the variable delay should be approximately $142 \mu \mathrm{s}$ for the EM pulse to encounter the acoustic wave at the middle of the 1 inch thick agar.

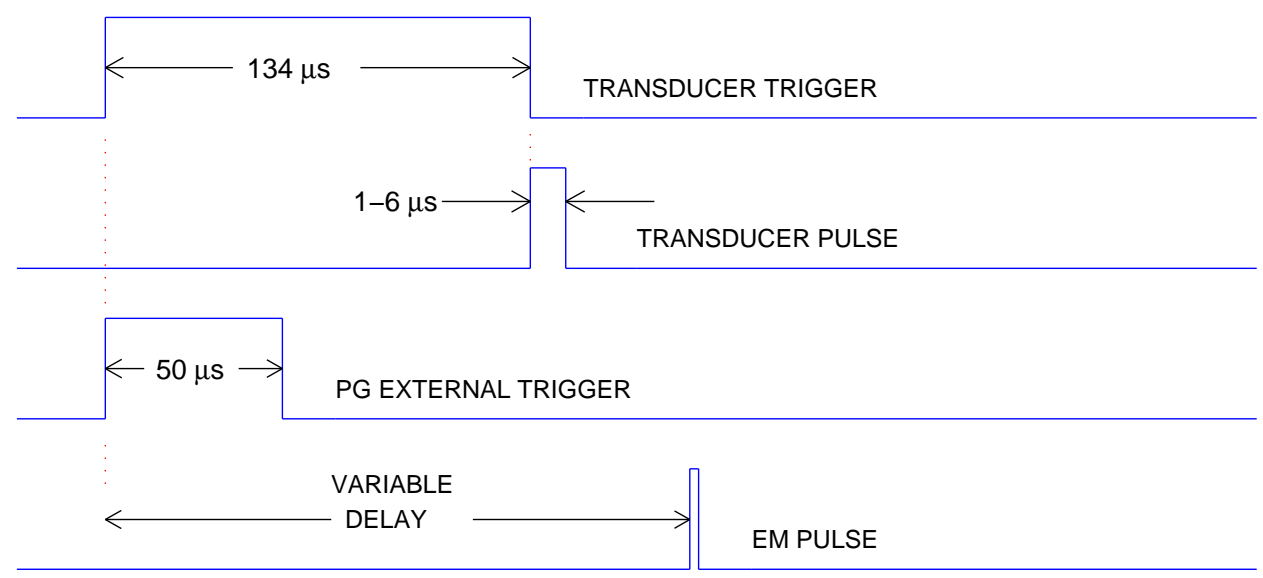

Figure 16: Schematic diagram of the signal timing. PG refers to the Pulse Generator.

\footnotetext{
${ }^{1}$ More accurately, the Delay Time is the time between output of the SYNCH pulse and output of the EM pulse. There is a fixed propagation delay of $60 \mathrm{~ns}$ between the External Trigger and SYNCH pulses.
} 


\section{EM/ACOUSTIC REFLECTIONS IN AGAR}

In these experiments we make TDR measurements with and without adding acoustic pulses. Each waveform plotted is the average of 4096 measurements. The waveforms with and without the acoustic pulse are subtracted to look for differences that may be due to a reflection of the EM pulse from the acoustic wave front. Radiation absorbing tiles and $125 \Omega$ resistive termination were used in the experiments reported in this section.

\subsection{Experiments With Different Delays}

We next perform TDR experiments with an agar target and acoustic waves. We vary the delay time to sample a range of possible positions of the acoustic wave in the agar at the time of incidence of the EM wave. The speed of the EM pulse in the agar is estimated to be $3.4 \times 10^{7} \mathrm{~m} / \mathrm{s}$, while the speed of sound in the agar was measured to be $1450 \mathrm{~ms} / \mathrm{s}$. Figure 17 plots the positive portions of the transducer receiver signals for the different delay times that were used. Time $t=0$ indicates the time at which the EM wave is launched in the antenna. As seen in Fig. 17, the transducer receiver signals are shifted relative to the launch times $(t=0)$, according to the different delay times used.

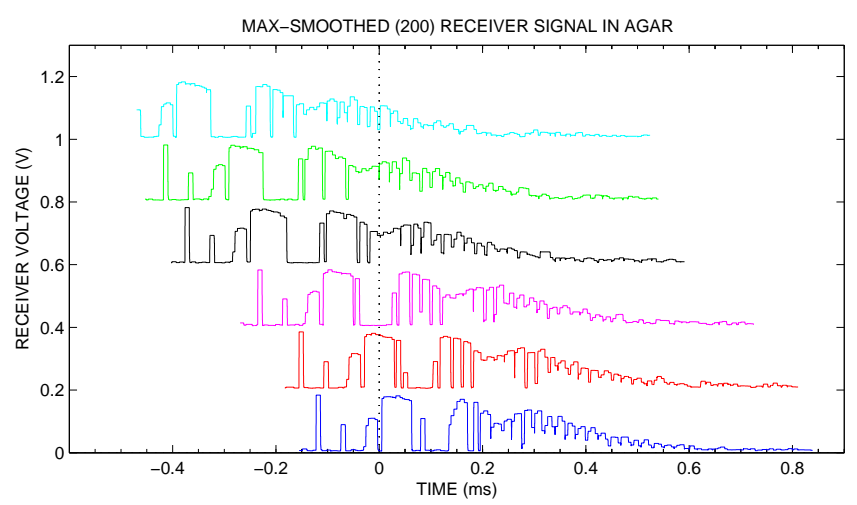

Figure 17: The positive portion of the min/max smoothed signal from the transducer receiver circuit for different delay times: $540 \mu \mathrm{s}$ (cyan), 435 (green), 395 (black), 255 (magenta), 174 (red), and $142 \mu \mathrm{s}$ (blue). The time axis is measured relative to the launch time of the EM pulse $(\mathrm{t}=0)$. 
The TDR measurements for the six different delay times, with and without acoustic waves, are plotted in the upper half of Figure 18. Because the differences among the measurements are small, all twelve curves appear to be coincident at this scale. The initial reflection of the EM pulse off the agar surface is indicated by the first negative swing in the TDR signals at approximately $26.5 \mathrm{~ns}$ (top plot). The first reflection of the EM pulse from the acoustic wave is expected to occur shortly after this time. Because of reflections from other structures in the antenna, reflections of the EM wave off the acoustic wave structure, if they exist, are expected to be small in comparison. For this reason we plot a subtracted signal in the lower half of Figure 18. The subtracted signal is the difference between TDR measurements with and without an acoustic wave in the agar target. Ideally, the plot of the subtracted signals would indicate only those events related to the reflection of the EM pulse from the acoustic wave. However, there appear to be three main features visible in the subtracted signal: the large spikes associated with the rise and fall of the input pulse, a smaller pair of spikes that occur approximately 19 ns later, and the presence of long-term oscillations (60-180 ns).

091704 data. TDR. 4096 ave.
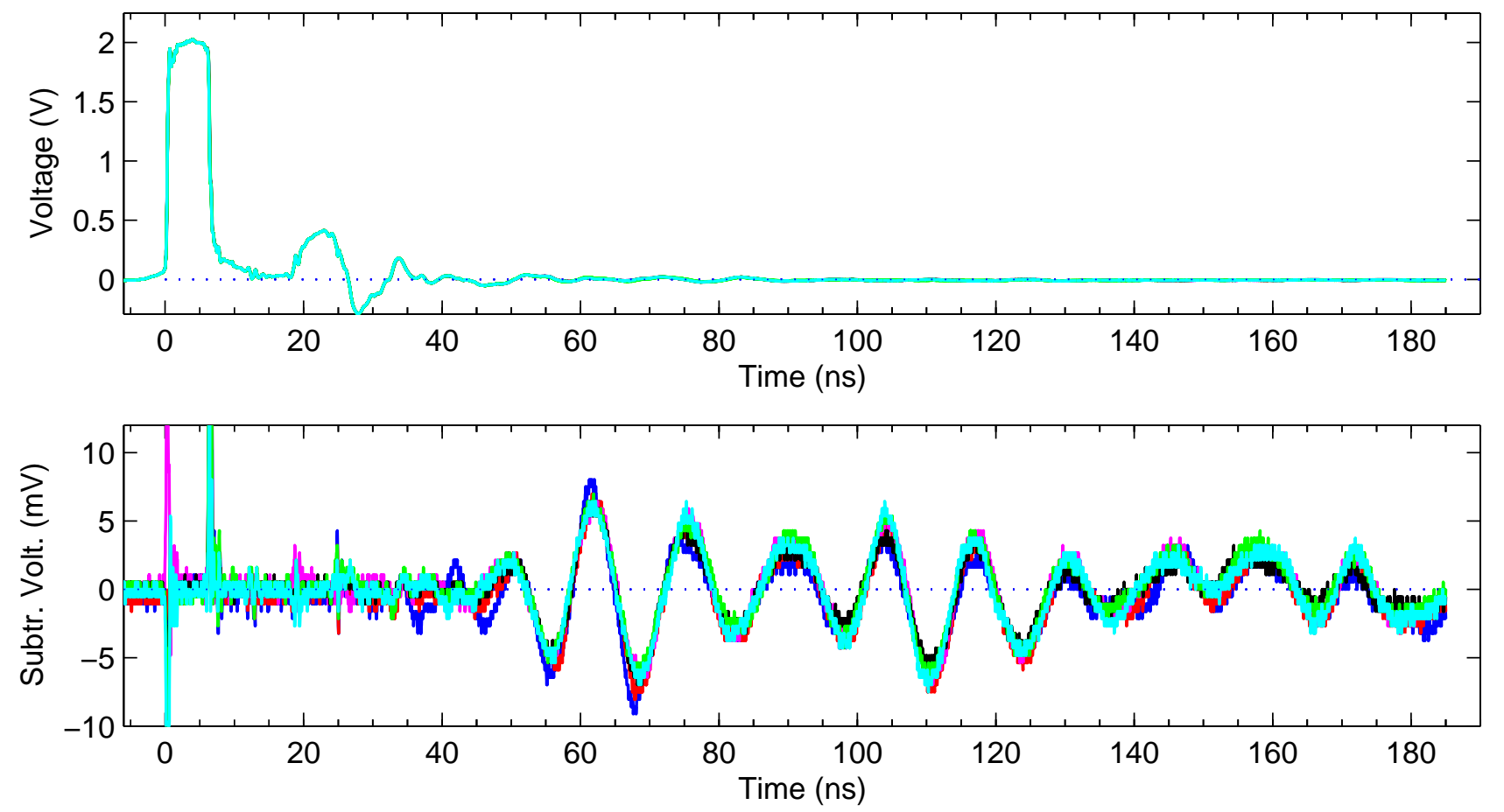

Figure 18: The top figure plots the TDR measurements for the different delay times with and without acoustic signals. There is little difference among the measurements, thus they appear as a single curve. The lower plot shows the subtracted signals (measurement without acoustic pulse minus measurement with acoustic pulse) for the different delay times: $540 \mu \mathrm{s}$ (cyan), 435 (green), 395 (black), 255 (magenta), 174 (red), and $142 \mu$ s (blue). Note that the units of the lower plot is in millivolts. 
Input pulse jitter. In Figure 19 we take a closer look at the large spikes in the subtracted signal that are associated with the rise and fall of the input pulse. Without jitter, the subtracted voltage should be zero for the duration of the pulse width. The spikes in the subtracted signals that occur at the leading and trailing ends of the input pulse may be due to jitter in the pulse width or jitter in the synchronization signal that triggers the oscilloscope. There seems to be a correlation in the negative and positive spikes; a negative spike in the leading edge of the pulse is generally followed by a positive spike in the trailing edge of the input pulse subtracted signal. This suggest that jitter in the synchronization signal may be the main source of the discrepancies in the subtracted signal. If the timing of the synchronization signal varies a little bit, the time at which the input pulses are sampled by the oscilloscope will vary a little bit. This would produce spikes at the edges of the input pulse when the two signal are subtracted, since they would be slightly offset.

091604 data. TDR. 4096 ave.
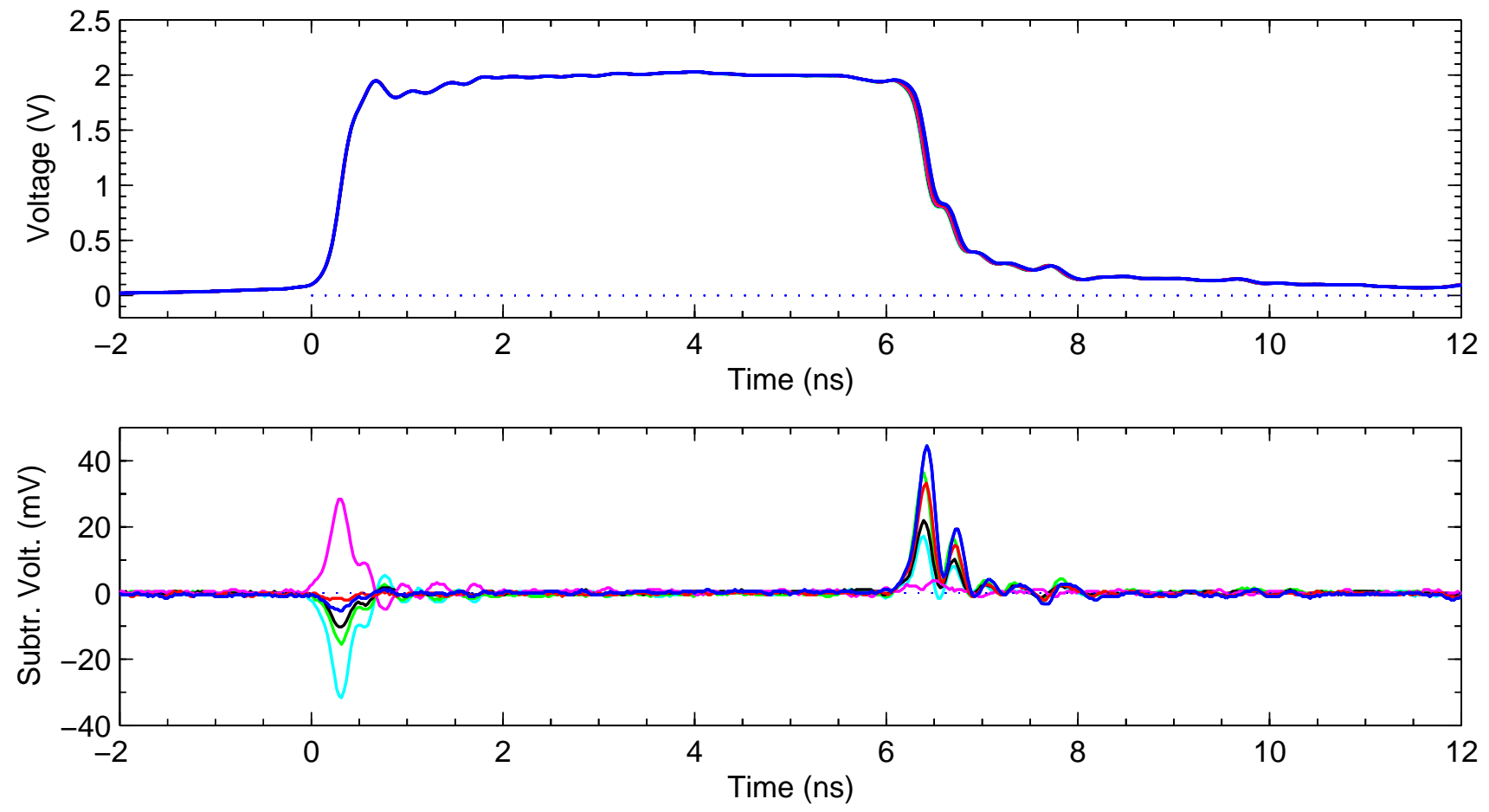

Figure 19: This figure gives an expanded view around the time that the input pulse arrives. The top figure plots the TDR measurements for the different delay times with and without acoustic signals. There is little difference among the measurements, thus they appear as a single curve. The lower plot shows the subtracted signals (measurement without acoustic pulse minus measurement with acoustic pulse) for the different delay times: $540 \mu$ s (cyan), 435 (green), 395 (black), 255 (magenta), 174 (red), and $142 \mu$ s (blue). 
Events in the vicinity of the EM/Acoustic interaction. Figure 20 also provides a closer look at the above plot in the time region where we expect the EM and acoustic waves to be coincident. The speed of the EM pulse in the agar is $3.4 \times 10^{7} \mathrm{~m} / \mathrm{s}$, so the EM pulse should encounter the acoustic wave at the 27-28 ns mark. We do, in fact, see voltage spikes in the subtracted signal at $25 \mathrm{~ns}$. If the events at the $25 \mathrm{~ns}$ mark were due to EM reflections from the acoustic wave, we would, because of the different delay times, expect these reflection events occur at slightly different times. We can also see from Fig. 20 that there are voltage spikes in the subtracted signal that occur approximately $6 \mathrm{~ns}$ earlier, at the $19 \mathrm{~ns}$ mark. The time at which these pairs of spikes occur, $19 \mathrm{~ns}$ after the leading and trailing edges of the input pulse, and the temporal separation of these spikes (6 ns) suggest that they are probably related to the reflection of the input pulse at the feed of the antenna. The variability in the input pulse synchronization is also seen in its reflection at the feed. We are observing the input pulse jitter again, although it has been attenuated.

091604 data. TDR. 4096 ave.
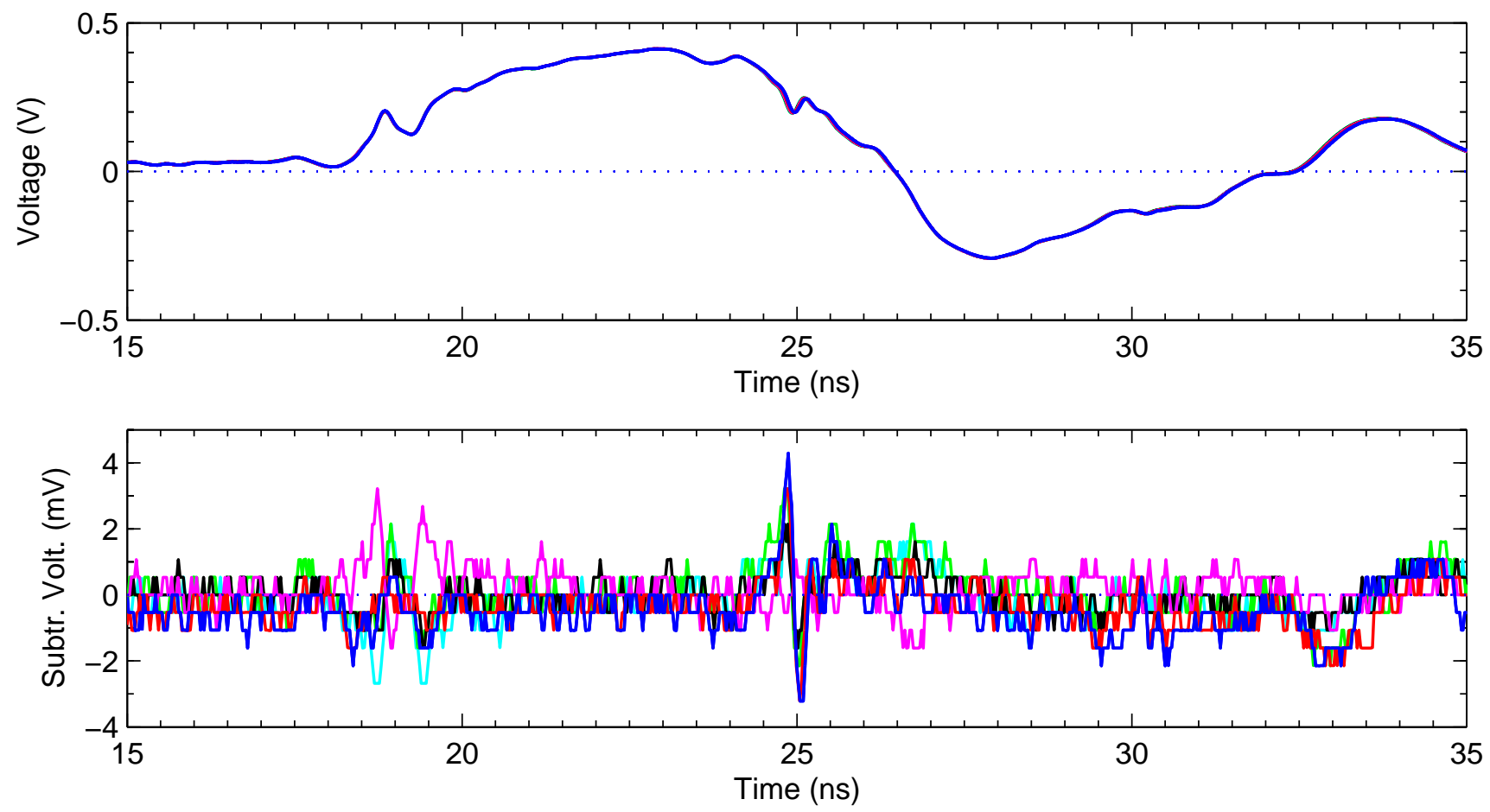

Figure 20: This figure gives an expanded view around the time that the input pulse first encounters the acoustic wave. The top figure plots the TDR measurements for the different delay times with and without acoustic signals. There is little difference among the measurements, thus they appear as a single curve. The lower plot shows the subtracted signals (measurement without acoustic pulse minus measurement with acoustic pulse) for the different delay times: $540 \mu$ s (cyan), 435 (green), 395 (black), 255 (magenta), 174 (red), and $142 \mu$ s (blue).

\subsection{Experiment With Different Pulse Widths}

To further explore the phenomena that we observed in the above data, another set of measurements was performed where the delay time was kept constant, but the width of the input pulse was varied (Figure 21). 

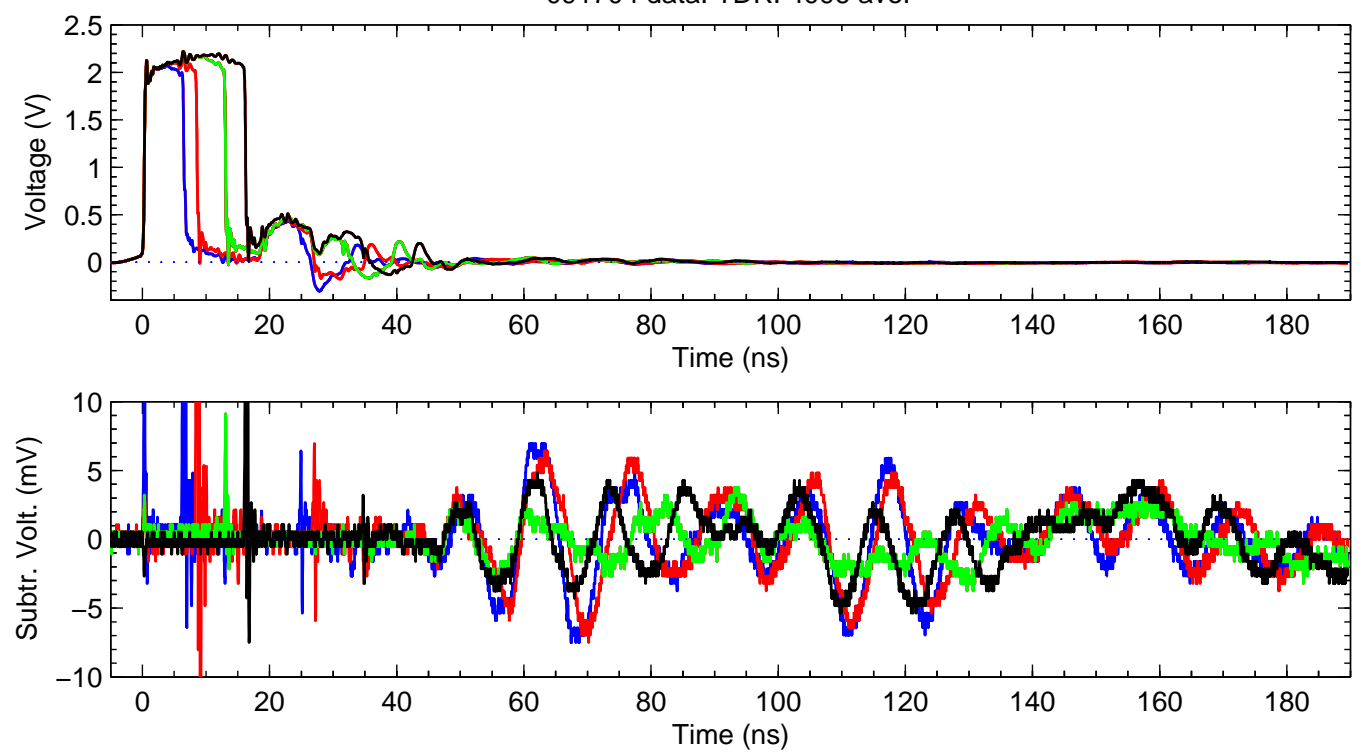

Figure 21: The top figure plots the TDR measurements for the different input pulse widths, with and without acoustic signals. The lower plot shows the subtracted signals (measurement without acoustic pulse minus measurement with acoustic pulse) for the different pulse widths: 7 (blue), 9 (red), 13 (green), and 16 ns (black). Note that the units of the lower plot is in millivolts.

Figure 22 expands the view around the input pulse. Again we can see voltage spikes in the subtracted view (lower plot) associated with the leading and trailing edges of the input pulses. However, in the case of the 13 ns pulse (green curve), timing of the acoustic and non-acoustic input pulses is closely matched and the subtracted signal has only very small spikes in the vicinity of the leading and trailing edges.
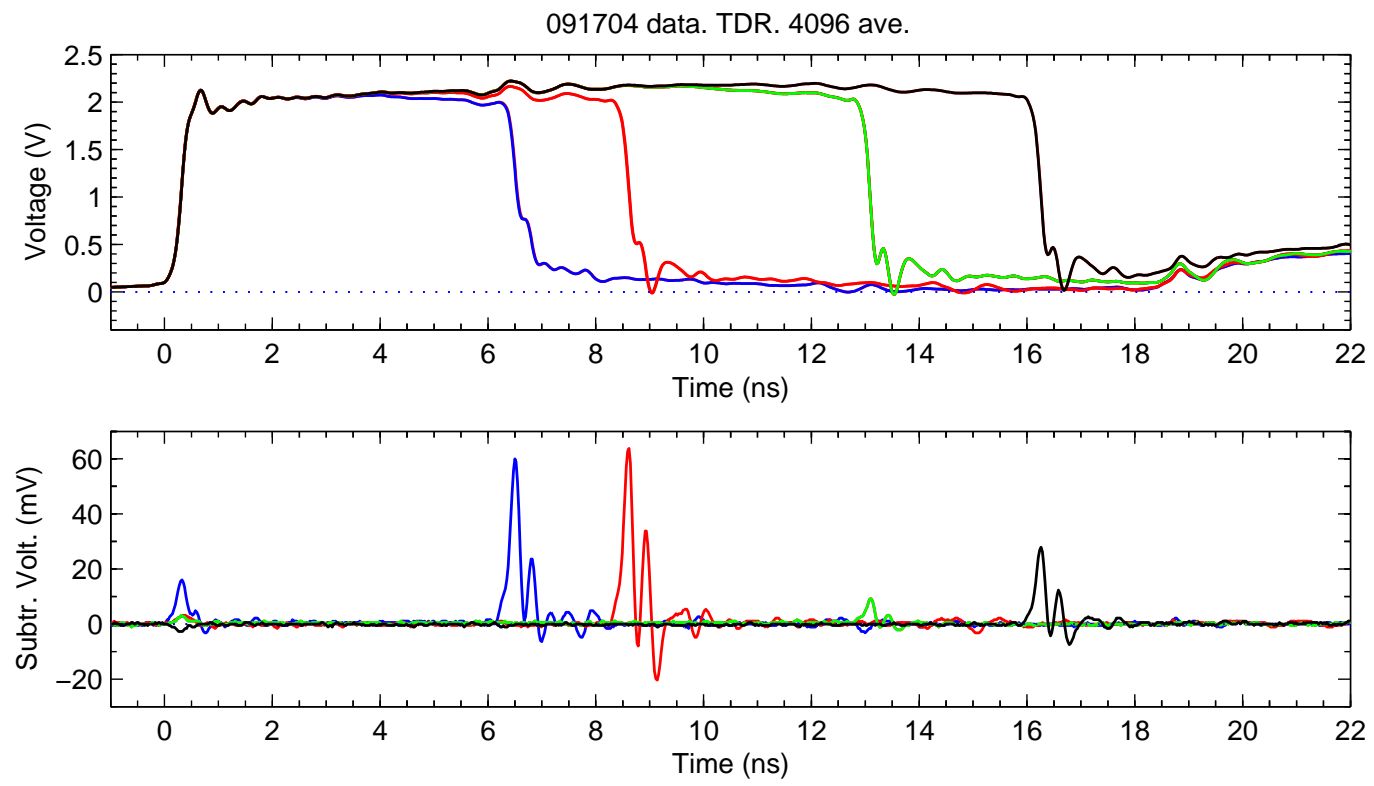

Figure 22: An expanded view of the input pulses and the subtracted waveforms. The top figure plots the TDR measurements for the different input pulse widths, with and without acoustic signals. The lower plot shows the subtracted signals (measurement without acoustic pulse minus measurement with acoustic pulse) for the different pulse widths: 7 (blue), 9 (red), 13 (green), and $16 \mathrm{~ns}$ (black). Note that the units of the lower plot is in millivolts. 
Figure 23 expands the view in Fig. 21 in the vicinity of the EM/acoustic wave reflections. In Fig. 18 above, we saw that the events at the 25 ns mark were coincident even though we had different delay times. In Fig. 23 (lower plot) we can see that these events are no longer coincident when the pulse width is varied (constant delay time), further suggesting that these events are related to the input pulse jitter.
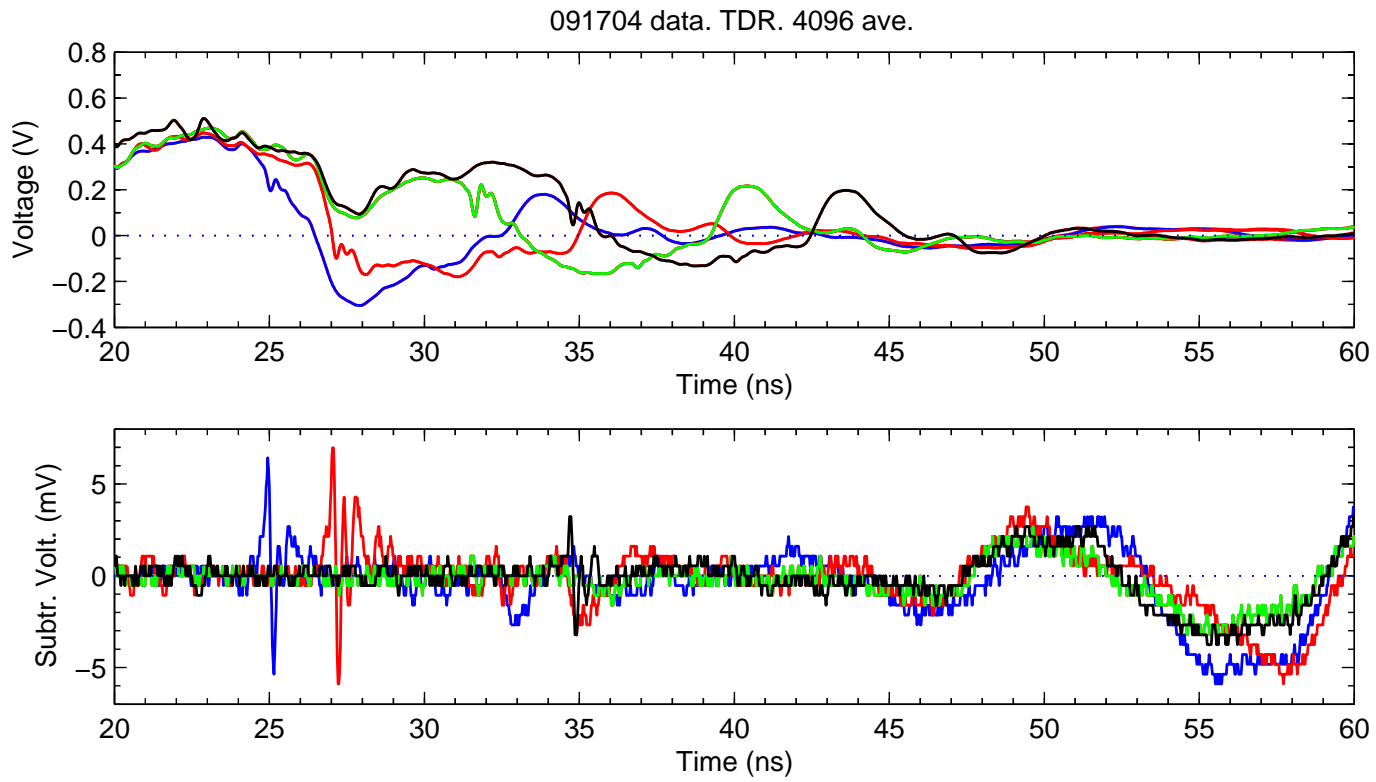

Figure 23: An expanded view in the vicinity of the EM/Acoustic interaction. The top figure plots the TDR measurements for the different input pulse widths, with and without acoustic signals. The lower plot shows the subtracted signals (measurement without acoustic pulse minus measurement with acoustic pulse) for the different pulse widths: 7 (blue), 9 (red), 13 (green), and 16 ns (black). Note that the units of the lower plot is in millivolts.

If we plot the times at which these events occur as a function of the pulse width, we see a strong linear relationship (Fig. 24). In this plot, there is no data point corresponding to the $13 \mathrm{~ns}$ pulse width, since there was no clearly observable voltage event in the vicinity of the expected time of the EM/acoustic wave reflection. This is further evidence that the observed voltage events are related to the input pulse jitter, since there was very little jitter in the 13 ns input pulse (Fig. 22).

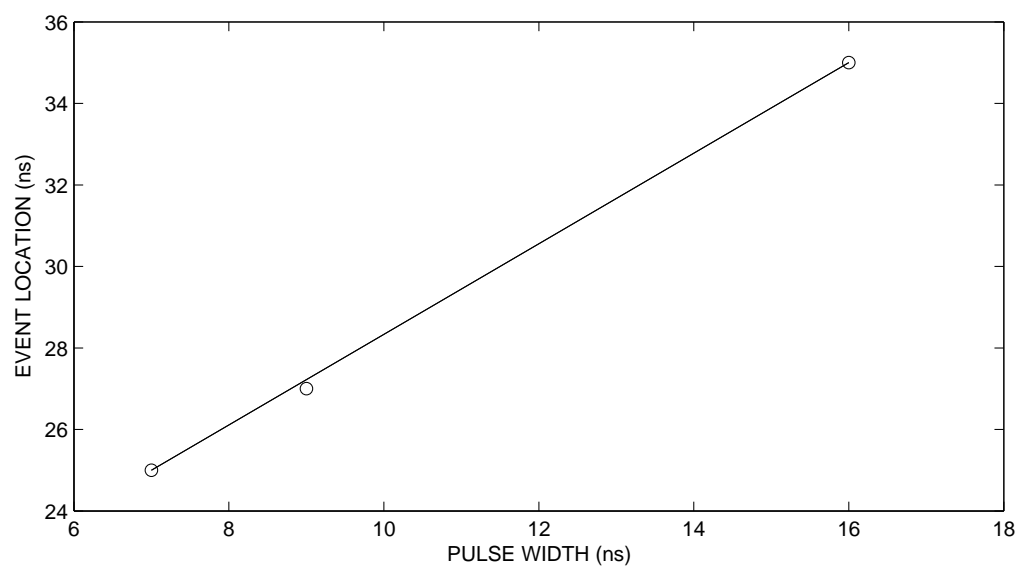

Figure 24: Location of the spikes in the subtracted signal (Fig. 23) as a function of the input pulse width. 


\subsection{Experiment With Thicker Agar}

The time for the EM pulse to traverse the 1 in. thick agar is roughly $0.75 \mathrm{~ns}$. This makes it difficult to separate the EM reflection at the front of the agar from the EM reflection at the acoustic wavefront. Figure 25 plots the results of measurements made with a 4 inch thick agar target. As in previous experiments we take data with and without an acoustic wave in the agar, as well as recording a third data set, also without an acoustic signal. When plotted together all three waveforms appear indistinguishable (Fig. 25, top plot). If one of the non-acoustic data sets is subtracted from the acoustic data set we get results that are similar to what we have already seen (Fig. 25, bottom plot, blue curve). Subtracting one non-acoustic data set from the other provides a control for comparison to the acoustic data (Fig. 25, bottom plot, red curve).

Both the acoustic and the control wave forms in Fig. 25 (lower plot) have spikes at 19 and 25 ns, further evidence that this is a remnant of the input pulse and not an acoustic reflection event. There seems to be jitter in the input pulse peak voltage. Both sets of subtracted data are nonzero during the input pulse time.

102104 data. TDR. 4096 ave.
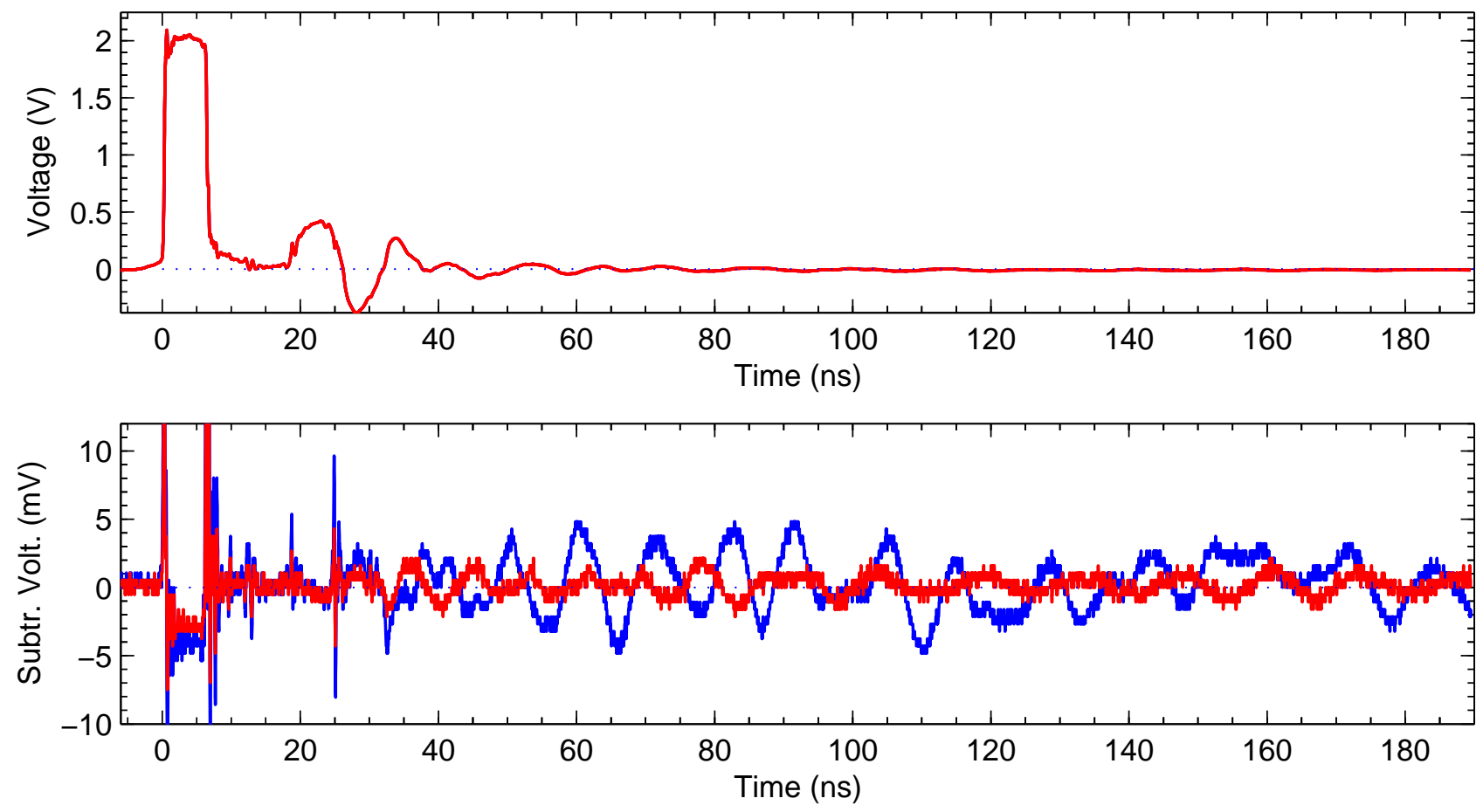

Figure 25: TDR and subtracted data with a 4 inch thick agar target. In the lower plot, the blue curve is the subtracted data for acoustic minus no-acoustic measurements. The red curve is the subtracted data for two no-acoustic measurements. 


\subsection{EM/Acoustic Reflections Without Agar}

One feature of the subtracted TDR signals that is consistently observed is the presence of long term oscillations. These oscillations are present in the control (Fig. 25, bottom plot, red curve), although the amplitude of the oscillations is much reduced. In order to explore the possiblity that these oscillations are an artifact of the experiment and not related to EM/acoustic reflections, we measurements, as above, but without an agar target in the antenna. In this experiment, the transducer is mounted through a styrofoam block (2.5" wide, 10" high, and 1" thick) that acts as a support for the transducer. The back of the styrofoam block is flush with the pyramidal radiation absorbing tiles at the aperture of the antenna. The front surface of the transducer is flush with the front surface of the styrofoam block, about 1.5" from the back of the antenna, and 5.25" above the ground plane. The transducer is pointed toward the feed of the antenna. The transducer wire feeds through a small opening in the panel of radiation absorbing tiles.

Figure 26 plots subtracted TDR data for the following experimental conditions. The subtracted data plotted with the red curve was obtained in a manner similar to the acoustic experiments with an agar target, that is, TDR data with no acoustic wave is subtracted from TDR data taken with an acoustic wave. As is usually done, the nonacoustic data is taken with the timing circuit disconnected from the transducer circuit so that no trigger events are sent to the transducer drive circuit. The timing circuit is externally triggering the signal generator (this data was taken with a $180 \mu$ s delay time). As the red curve illustrates, there are strong long-term oscillations similar to what has been seen with an agar target.

The data plotted with the blue curve is the subtracted data of two nonacoustic TDR measurements, where the nonacoustic experimental conditions are achieved in two different ways. In one case the timing circuit is disconnected from transducer circuit, as above. In the second case the transducer drive circuit remains connected to the timing circuit, but there is no power driving the timing circuit and the Signal Generator is set at a $100 \mathrm{~Hz}$ repetition rate. As seen in Figure 26, the nonacoustic subtracted data shows similar long-term oscillations as the acoustic/nonacoustic subtracted data.

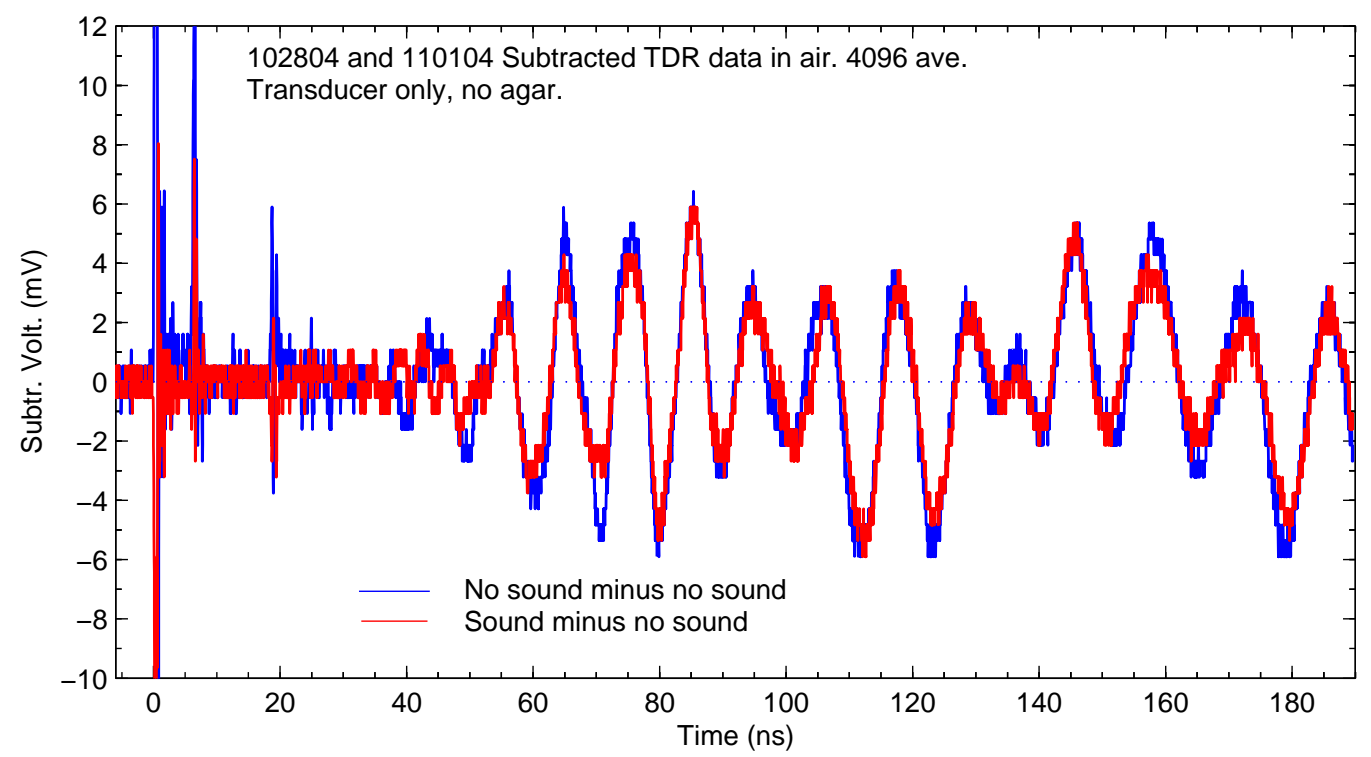

Figure 26: Subtracted signals for TDR data taken with no agar target. The blue curve is the subtracted signal from two nonacoustic data sets, taken under different experimental conditions. The red curve is the subtracted signal from acoustic and nonacoustic data sets without an agar target. 
For comparison purposes, Figure 27 plots the subtracted data obtained under the two nonacoustic experimental conditions along with acoustic/nonacoustic subtracted data obtained with an agar target in the antenna. The data in the lower plot of Figure 27 show that the magnitude of the long term oscillations are similar for both data sets, although the frequency of oscillations is greater for the data from the agar target.

102104 data. TDR. 4096 ave.
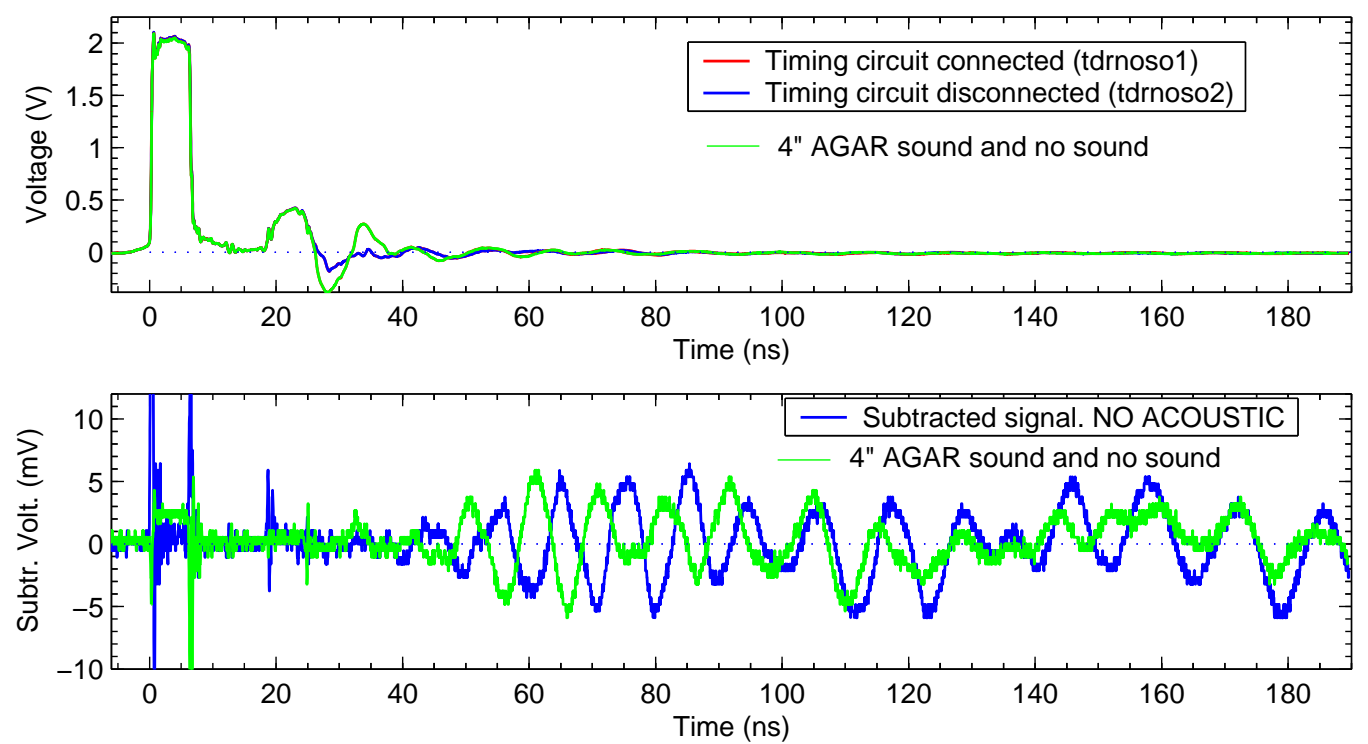

Figure 27: TDR measurements with and without an agar target in the antenna. The blue curve in the lower plot is the subtracted signal from two nonacoustic TDR data sets, taken under different experimental conditions. The green curve in the lower plot is the subtracted signal from acoustic and nonacoustic data sets with an agar target.

We have previously seen, with the agar target experiments, that the long term oscillations in the subtracted data remain constant whether the timing circuit is externally driving the signal generator or the signal generator is at a fixed repetition rate (data not shown in this report). The results of these experiments would seem to indicate that the main features of the long-term oscillations in the subtracted data are not the result of acoustic events, but an artifact of the transducer/timer circuitry interacting with the electromagnetic signal in the case when the transducer circuit is connected to the timing circuit. 


\section{COMPUTATIONAL STUDIES}

As the above experimental results indicate, we are unable to detect reflections of the microwave EM pulse from the acoustic wave in the agar material. Previous computational work illustrated the type of reflections that would be observed with pressure dependent Debye parameters [1]. However, the parameter values used in that study were chosen in order to produce a measurable effect and are not representative of a particular material. In this section we seek to obtain an estimate for the pressure dependence of the dielectric response of agar and then turn again to computational simulations, this time to attempt to explain the failure to detect EM reflections in our experimental wave guide.

\subsection{Dielectric Properties of Agar}

Miura et al.[13] model the permittivity of agar using the Havriliak-Negami equation

$$
\epsilon-\epsilon_{\infty}=\frac{\epsilon_{s m}-\epsilon_{\infty}}{\left(1+\left(i \omega \tau_{m}\right)^{\beta_{m}}\right)^{\alpha_{m}}}+\frac{\epsilon_{s h}-\epsilon_{\infty}}{1+\left(i \omega \tau_{h}\right)^{\beta_{h}}},
$$

where $\omega$ is the frequency $(\mathrm{Hz})$, and the subscripts $m$ and $h$ refer to the middle and high frequency regimes, respectively. They find the following parameter values for agar (1\% by weight) $\epsilon_{s m}=9.0, \tau_{m}=1.82 \times 10^{-8}$, $\alpha_{m}=0.94, \beta_{m}=1.0, \epsilon_{s h}=77.0, \tau_{h}=8.51 \times 10^{-12}, \beta_{h}=1.0$, and $\epsilon_{\infty}=5.0$.

Not too surprisingly, the permittivity of agar closely resembles, in the high frequency limit, that of water, which has a single molecular relaxation term [8]

$$
\epsilon-\epsilon_{\infty}=\frac{\epsilon_{s}-\epsilon_{\infty}}{1+(i \omega \tau)}
$$

where $\epsilon_{s}=78.47$ is the static permittivity, $\epsilon_{\infty}=5.2$ is the permittivity in the high frequency limit, and $\tau=8.27 \times 10^{-12}$ os the relaxation time for molecular orientation.

\subsection{Pressure Dependence of the Permittivity of Water}

Since we are unable to find data in the literature that would allow us to estimate the pressure dependence of agar, we can find similar values for water, which it closely resembles. In this section we present the results of a literature search on the pressure dependence of the Debye parameters for water.

Following the work of Albanese et al.[1] we first approximate the pressure dependence of the Debye parameters to be a linear perturbation about the parameter values at atmospheric pressure

$$
\begin{aligned}
\tau(p) & =\tau_{0}+\kappa_{\tau} p \\
\epsilon_{s}(p) & =\epsilon_{s, 0}+\kappa_{s} p \\
\epsilon_{\infty}(p) & =\epsilon_{\infty, 0}+\kappa_{\infty} p,
\end{aligned}
$$

where $p$ is the pressure and $\kappa_{\tau}, \kappa_{s}$, and $\kappa_{\infty}$ are the coefficients of pressure for $\tau, \epsilon_{s}$, and $\epsilon_{\infty}$, respectively.

We were unable to find any data relevant to the pressure dependence of $\epsilon_{\infty}$ in our literature search. However, we did find information on $\kappa_{s}$ and $\kappa_{\tau}$, which we present below.

\subsection{Estimate for $\kappa_{s}$}

There are a number of sources in the literature from which one can obtain estimates for the pressure dependence of $\epsilon_{s}$. We can obtain a rough estimate for $\left.\left(\partial \epsilon_{s} / \partial P\right)\right|_{T}$ from published values in the CRC 
Handbook ([8], pg. 6-15). Linearly interpolating from data at 0.1 and $1 \mathrm{MPa}$ and $295^{\circ} \mathrm{K}$ and $300^{\circ} \mathrm{K}$, we get room temperature $T=298^{\circ} \mathrm{K}$ values of $\epsilon_{s}=78.47$ and 78.50 at $P=0.1$ and $1.0 \mathrm{MPa}$, respectively. These $\epsilon_{s}$ values provide a crude estimate of $\left.\left(\partial \epsilon_{s} / \partial P\right)\right|_{T}=\kappa_{s}=3.33 \times 10^{-2} \mathrm{MPa}^{-1}=3.33 \times 10^{-8} \mathrm{~Pa}^{-1}$ at room temperature and atmospheric pressure $(P=0.101325 \mathrm{MPa})$.

Fernandez et al.[9] determined that $\epsilon_{s}=78.41$ and $\left.\left(\partial \epsilon_{s} / \partial P\right)\right|_{T}=3.74 \times 10^{-2} \mathrm{MPa}^{-1}=3.74 \times 10^{-8} \mathrm{~Pa}^{-1}$ at $T=298.144^{\circ} \mathrm{K}$ and atmospheric pressure.

Floriano and Nascimento [10] extend the work of Bradley and Pitzer [6] to more accurately fit the static dielectric constant $\epsilon_{s}$ to published data [8] at low temperatures and high pressures (0-2000 MPa). Their model for the static dielectric constant at room temperature $\left(298^{\circ} \mathrm{K}\right)$ as a function of pressure is given by

$$
\epsilon_{s}(P)=\epsilon_{R}+a_{0} \ln \left(\frac{a_{1}+P}{a_{1}+P_{R}}\right)
$$

where $P_{R}$ is a reference pressure, in their case $10 \mathrm{MPa}, P$ is the pressure in $\mathrm{MPa}$, and $\epsilon_{R}$ represents the value of the static dielectric constant at the reference pressure. At $T=298^{\circ} \mathrm{K}, a_{0}=14.1113, a_{1}=341.5902$ $\mathrm{MPa}$, and $\epsilon_{R}=78.85$.

Since we are interested in a linearized model (7.3), we can linearize (7.4) about $P_{0}=0.1 \mathrm{MPa}$ by writing

$$
\begin{aligned}
\epsilon_{s}(P) & \approx \epsilon_{s}\left(P_{0}\right)+\left.\frac{d \epsilon_{s}}{d P}\right|_{P_{0}} \Delta P \\
& =\epsilon_{s}\left(P_{0}\right)+\frac{a_{0}}{a_{1}+P_{0}}\left(P-P_{0}\right) \\
& =\left(\epsilon_{s}\left(P_{0}\right)-\frac{a_{0} P_{0}}{a_{1}+P_{0}}\right)+\frac{a_{0}}{a_{1}+P_{0}} P \\
& =\epsilon_{s}+\kappa_{s} P,
\end{aligned}
$$

where

$$
\epsilon_{s}=\epsilon_{R}+a_{0} \ln \left(\frac{a_{1}+P_{0}}{a_{1}+P_{R}}\right)-\frac{a_{0} P_{0}}{a_{1}+P_{0}}
$$

and

$$
\kappa_{s}=\frac{a_{0}}{a_{1}+P_{0}}
$$

Choosing $P_{0}=1$ atmosphere $=0.101 \mathrm{MPa}$, gives $\epsilon_{s}=78.44$ and $\kappa_{s}=4.13 \times 10^{-2} \mathrm{MPa}^{-1}=4.13 \times 10^{-8}$ $\mathrm{Pa}^{-1}$.

\subsection{Estimate for $\kappa_{\tau}$}

Using data, Shcherbakov [16] estimates the pressure dependence of $\tau$ to be

$$
\tau(P)=\tau_{0}-\kappa_{\tau}^{*} P^{*} \quad(\mathrm{~s}),
$$

where $P^{*}$ is the pressure in $\mathrm{MPa}$,

$$
\kappa_{\tau}^{*}=\left(\frac{3.48 \times 10^{-12} \eta^{*}}{T}\right)=1.05 \times 10^{-14} \quad(\mathrm{~s} / \mathrm{MPa}),
$$

and $\eta^{*}$ is the viscosity in centipoise.

The above equations can be rewritten as

$$
\tau(P)=\tau_{0}-\kappa_{\tau} P \quad(\mathrm{~s}),
$$

where $P$ is the pressure in $\mathrm{Pa}$ and

$$
\kappa_{\tau}=1.05 \times 10^{-20} \quad(\mathrm{~s} / \mathrm{Pa})
$$




\subsection{Summary of Literature Search}

In summary, we have obtained estimates for the pressure dependence of the Debye parameters $\epsilon_{s}$ and $\tau$ for water from data available in the literature. No data was found for the pressure dependence of $\epsilon_{\infty}$. Based upon the measured frequency dependence of the permittivity of agar [13] we expect that the pressure-dependence of the Debye parameters for water would be a good first estimate for the pressure-dependent behavior of the permittivity of agar. Values for the pressure coefficients $\left(\kappa_{\tau}=-1.05 \times 10^{-20}\right.$ and $\left.\kappa_{s}=3.74 \times 10^{-8}\right)$ that were obtained from the literature are much smaller than the values used in previous simulations [1]. Therefore, we suggest that our experimental conditions are probably not sufficient to see reflections of the EM pulse from the pressure wave. (We estimate the magnitude of the pressure waves from the transducer to be on the order of $0.1 \mathrm{~Pa}$.)

The table below summarizes the Debye parameter values for water at atmospheric pressure and estimates from the literature for the (linear) coefficients of pressure.

Table 1: Parameter values for water at room temperature and atmospheric pressure

\begin{tabular}{l|c|c|c|c|c|} 
& $\tau_{0}[\mathrm{~s}]$ & $\epsilon_{s, 0}$ & $\epsilon_{\infty, 0}$ & $\kappa_{\tau}\left[\mathrm{s} \cdot \mathrm{Pa}^{-1}\right]$ & $\kappa_{s}\left[\mathrm{~Pa}^{-1}\right]$ \\
\hline CRC Handbook & $8.27 \times 10^{-12}$ & 78.47 & 5.2 & & $3.3 \times 10^{-8}$ \\
Fernandez et al. & & 78.41 & & & $3.74 \times 10^{-8}$ \\
Floriano and Nascimento & & 78.44 & & & $4.13 \times 10^{-8}$ \\
Shcherbakov & $8.2 \times 10^{-12}$ & & & $-1.05 \times 10^{-20}$ &
\end{tabular}

\subsection{Simulations}

We present numerical simulations for a Debye medium that is similar to water. For signal bandwidths in the microwave regime the dispersive properties of water are usually modeled by a Debye equation having a single molecular relaxation term. The mean values $\epsilon_{s, 0}, \epsilon_{\infty, 0}$, and $\tau_{0}$ for this Debye model are given by

$$
\begin{aligned}
& \epsilon_{s, 0}=78.44 \text { (relative static permittivity), } \\
& \epsilon_{\infty, 0}=5.2 \text { (relative high frequency permittivity) } \\
& \tau_{0}=8.2 \times 10^{-12} \text { seconds, } \\
& \sigma \quad=1 \times 10^{-5} \text { mhos } / \text { meter }
\end{aligned}
$$

where $\sigma$ is the conductivity of water.

Following the notation in [3] we represent the pressure dependent parameters $\epsilon_{s}, \epsilon_{\infty}$, and $\tau$ as a mean value plus a perturbation that is proportional to the pressure, with the coefficients of pressure designated as $\kappa_{s}$, $\kappa_{\infty}$ and $\kappa_{\tau}$, respectively. Based upon the above literature search and analysis, we estimate the following values for the coefficients of pressure dependence: $\kappa_{s}=3.74 \times 10^{-8} \mathrm{~Pa}^{-1}$ and $\kappa_{\tau}=-1.05 \times 10^{-20} \mathrm{~s} / \mathrm{Pa}$, with $\kappa_{\infty}$ undetermined. As demonstrated in [3], the acoustic reflection is not sensitive to changes in either $\kappa_{\infty}$ or $\kappa_{\tau}$, but is sensitive to changes in $\kappa_{s}$. Based on these observations and the low value of $\kappa_{\tau}$, we chose the following values for our simulations

$$
\begin{aligned}
& \kappa_{s}=3.74 \times 10^{-8} \mathrm{~Pa}^{-1}, \\
& \kappa_{\infty}=0.0, \\
& \kappa_{\tau}=0 \mathrm{~s} / \mathrm{Pa} .
\end{aligned}
$$




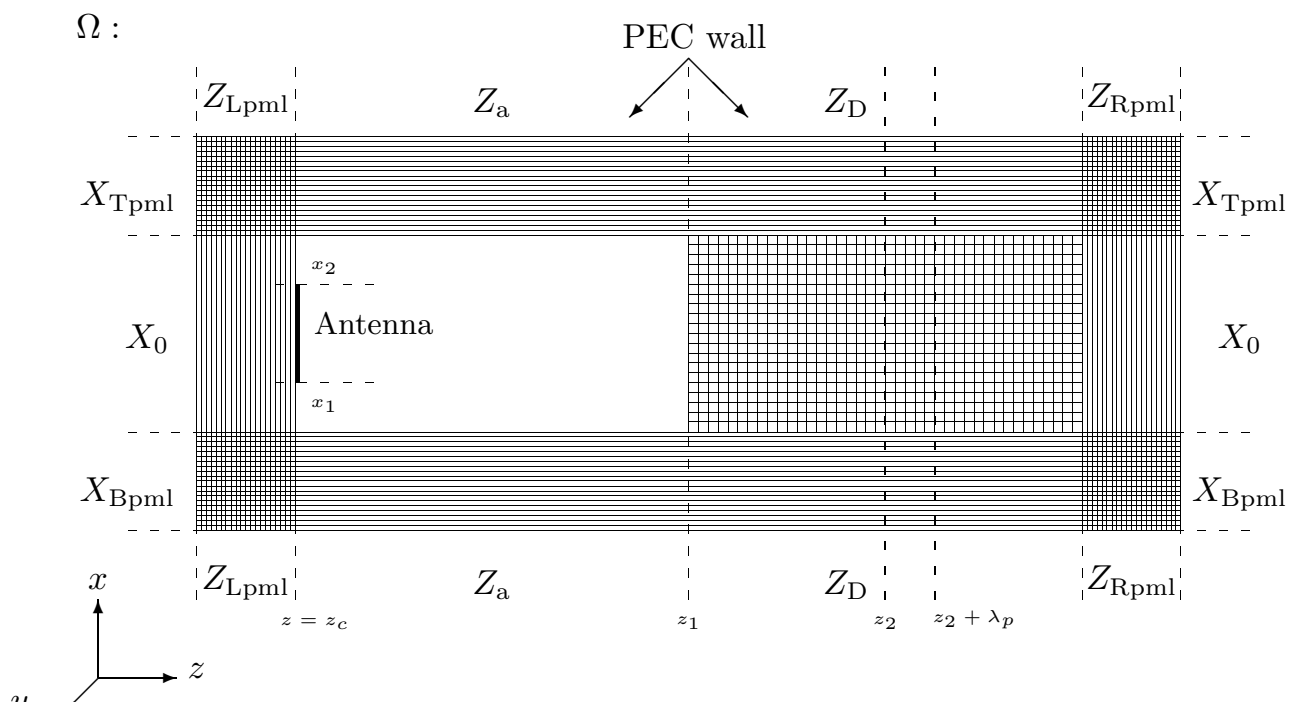

Figure 28: PML layers surrounding the domain of interest.

The computational domain is similar to that of [3] and is defined as follows. We take $X_{0}=(0,0.1)$, $Z_{\mathrm{a}}=(0,0.15)$ and $Z_{\mathrm{D}}=(0.15,0.2)$. The number of nodes along the $z$-axis is taken to be 320 and the number of nodes along the $x$-axis is taken to be 160 . The spatial step size in both the $x$ and $z$ directions is $\Delta x=\Delta z=h=0.1 / 160$. From the CFL condition with the Courant number $\eta_{\mathrm{CN}}=1 / 2$ we obtain the time increment to be $\Delta t \approx 1.0417$ pico seconds. The central frequency of the input source as described in ( 7.19$)$ is $3.0 \mathrm{GHz}$ and based on the speed of light in air, $c_{0}=3 \times 10^{8} \mathrm{~m} / \mathrm{s}$, we calculate the corresponding central wavelength to be $\lambda_{c}=\left(2 \pi c_{0}\right) / \omega=0.1$ meters. The source/observation antenna is half a wavelength long and is placed at $\left(x_{1}, x_{2}\right) \times z_{c}$, with $z_{c}=0, x_{1}=0.025$ and $x_{2}=0.075$. We use PML layers that are half a wavelength thick on all fours sides of the computational domain as shown in Figure 28 . The reflections of the electromagnetic pulse at the air-Debye interface and from the acoustic pressure wave are recorded at the center of the antenna $\left(x_{c}, z_{c}\right)$, with $x_{c}=0.05$, at every time step. The component of the electric field that is of interest here is the $E_{x}$ component of $\mathbf{E}$. Thus our "observations" for the reflected signals are

$$
\begin{aligned}
E\left(\mathbf{q}^{*}\right) & =\left\{E_{x}\left(n \Delta t, x_{c}, z_{c} ; \mathbf{q}^{*}\right)\right\}_{n=1}^{M} \\
\mathbf{q}^{*} & =\left(\epsilon_{s, 0}^{*}, \epsilon_{\infty, 0}^{*}, \tau_{0}^{*}, \sigma^{*}, \kappa_{s}^{*}, \kappa_{\infty}^{*}, \kappa_{\tau}^{*}\right)^{T}
\end{aligned}
$$

where $\mathbf{E}$ is the computed solution to Maxwell's equation as given in [3]. In particular, we use modified Maxwell's equations which govern the electric field $\mathbf{E}$ and the magnetic field $\mathbf{H}$ in a domain $\Omega$ with charge density $\rho$. Thus we first consider the system

$$
\left\{\begin{array}{l}
\text { (i) } \frac{\partial \mathbf{D}}{\partial t}+\mathbf{J}-\nabla \times \mathbf{H}=0, \text { in }(0, T) \times \Omega, \\
\text { (ii) } \frac{\partial \mathbf{B}}{\partial t}+\nabla \times \mathbf{E}=0, \text { in }(0, T) \times \Omega, \\
\text { (iii) } \nabla \cdot \mathbf{D}=\rho, \text { in }(0, T) \times \Omega, \\
\text { (iv) } \nabla \cdot \mathbf{B}=0, \text { in }(0, T) \times \Omega, \\
\text { (v) } \mathbf{E} \times \mathbf{n}=0, \text { on }(0, T) \times \partial \Omega, \\
\text { (vi) } \mathbf{E}(0, \mathbf{x})=0, \quad \mathbf{H}(0, \mathbf{x})=0, \text { in } \Omega .
\end{array}\right.
$$

We have $\mathbf{J}=\mathbf{J}_{c}+\mathbf{J}_{s}$, where $\mathbf{J}_{c}$ is a conduction current density and $\mathbf{J}_{s}$ is the source current density. We assume only free space (actually the antenna in our example) can have a source current, and $\mathbf{J}_{c}$ is only found 
in the dielectric material. The electromagnetic input source $\mathbf{J}_{s}$ has the form

$$
\begin{aligned}
\mathbf{J}_{s}(t, x, z) & =I_{\left(x_{1}, x_{2}\right)} \delta(z) \sin \left(\omega_{c}\left(t-3 t_{0}\right)\right) \exp \left(-\left[\frac{t-3 t_{0}}{t_{0}}\right]^{2}\right) \mathbf{i} \\
t_{0} & =\frac{1}{2 \pi \times 10^{9}} \mathrm{sec}, \omega_{c}=6 \pi \times 10^{9} \mathrm{rad} / \mathrm{sec}, f_{c}=3.0 \times 10^{9} \mathrm{~Hz} .
\end{aligned}
$$

The Fourier spectrum of this pulse has even symmetry about $3.0 \mathrm{GHz}$.

To the system (7.18), we add variables to account for the PML layers surrounding the computational domainsee [3] for complete details. Within the dielectric medium we have constitutive relations that relate the flux densities D, B to the electric and magnetic fields, respectively. We have

$$
\left\{\begin{array}{c}
\text { (i) } \quad \mathbf{D}=\epsilon_{0} \mathbf{E}+\mathbf{P} I_{D} \\
\left(\text { ii) } \quad \mathbf{B}=\mu_{0} \mathbf{H}\right. \\
\text { (iii) } \quad \mathbf{J}_{c}=\sigma \mathbf{E} I_{D}
\end{array}\right.
$$

In (7.20), $I_{D}$ denotes the indicator function on the Debye medium. Thus, $\mathbf{J}_{c}=0$ in air. The electric polarization $\mathbf{P}$ is given by

$$
\mathbf{P}=\mathbf{P}_{\mathrm{I}}+\mathbf{P}_{\mathrm{R}}=\epsilon_{0} \chi \mathbf{E}+\mathbf{P}_{\mathrm{R}}
$$

and hence the constitutive law $(7.20$, i) becomes

$$
\mathbf{D}=\epsilon_{0} \epsilon_{r} \mathbf{E}+\mathbf{P}_{\mathrm{R}}
$$

where $\epsilon_{r}=(1+\chi)$ is the relative permittivity of the dielectric medium. The Debye model is represented in differential form as

$$
\begin{aligned}
\tau \dot{\mathbf{P}}_{\mathrm{R}}+\mathbf{P}_{\mathrm{R}} & =\epsilon_{0}\left(\epsilon_{s}-\epsilon_{\infty}\right) \mathbf{E} \\
\mathbf{D} & =\epsilon_{0} \epsilon_{\infty} \mathbf{E}+\mathbf{P}_{\mathrm{R}}
\end{aligned}
$$

inside the dielectric, whereas $\mathbf{P}_{\mathrm{R}}=0, \epsilon_{\infty}=1$ in air, and $\epsilon_{\infty}=\epsilon_{r}$ inside the dielectric. We will henceforth denote $\mathbf{P}_{\mathrm{R}}$ by $\mathbf{P}$. In equation (7.23), the parameters $\epsilon_{s}$ and $\epsilon_{\infty}$ denote the static relative permittivity, and the value of permittivity for an extremely high $(\approx \infty)$ frequency field, respectively.

The pressure $p$ will be assumed to have the form

$$
p(t, z)=I_{\left(z_{2}, z_{2}+\lambda_{p}\right)}|p| \sin \left(\omega_{p}\left[t+\frac{z-z_{2}}{c_{p}}\right]\right),
$$

where $z_{2} \in Z_{\mathrm{D}}$ with $z_{1}<z_{2}$. The terms $\omega_{p}, \lambda_{p}|p|$ and $c_{p}$ denote the acoustic frequency, wavelength, pressure magnitude and speed respectively. The windowed acoustic pressure wave as defined in (7.24) has the parameter values $\omega_{p}=6.0 \pi \times 10^{5} \mathrm{rad} / \mathrm{sec}, c_{p}=1500 \mathrm{~m} / \mathrm{s}$, and thus, $\lambda_{p}=0.005$. Also $|p|=1 \mathrm{~Pa}$. The location of the pressure region is in the interval $\left(z_{2}, z_{2}+\lambda_{p}\right)=(0.175,0.18)$. Further details on these simulations can be found in [3].

Since only $\kappa_{s}|p|$ enters into these calculations, it is only the product that matters. In Figure 29 we plot the ratio of the maximum amplitude of the acoustic reflection to the maximum amplitude of the input source for different values of the product $\kappa_{s}|p|$. The other pressure coefficients, $\kappa_{\infty}$ and $\kappa_{\tau}$ are set to be zero. From our simulations we see that this ratio $\left|E_{A}\right| / \mid E_{\text {input }}$ is independent of the maximum amplitude of the source term, i.e., $\left|E_{A}\right|$ scales according to the value of $\left|E_{\text {input }}\right|$. We can see in Figure 29 that the ratio $\left|E_{A}\right| / \mid E_{\text {input }}$ is linearly dependent on the ratio $\kappa_{s}|p|$ for values larger than 10 . We note that $E=E\left(\kappa_{s}|p|\right)$ is not linear in $\kappa_{s}|p|$ even though we make the linear approximations of (7.3) for $\tau, \epsilon_{s}$ and $\epsilon_{\infty}$ as a function of $p$. 


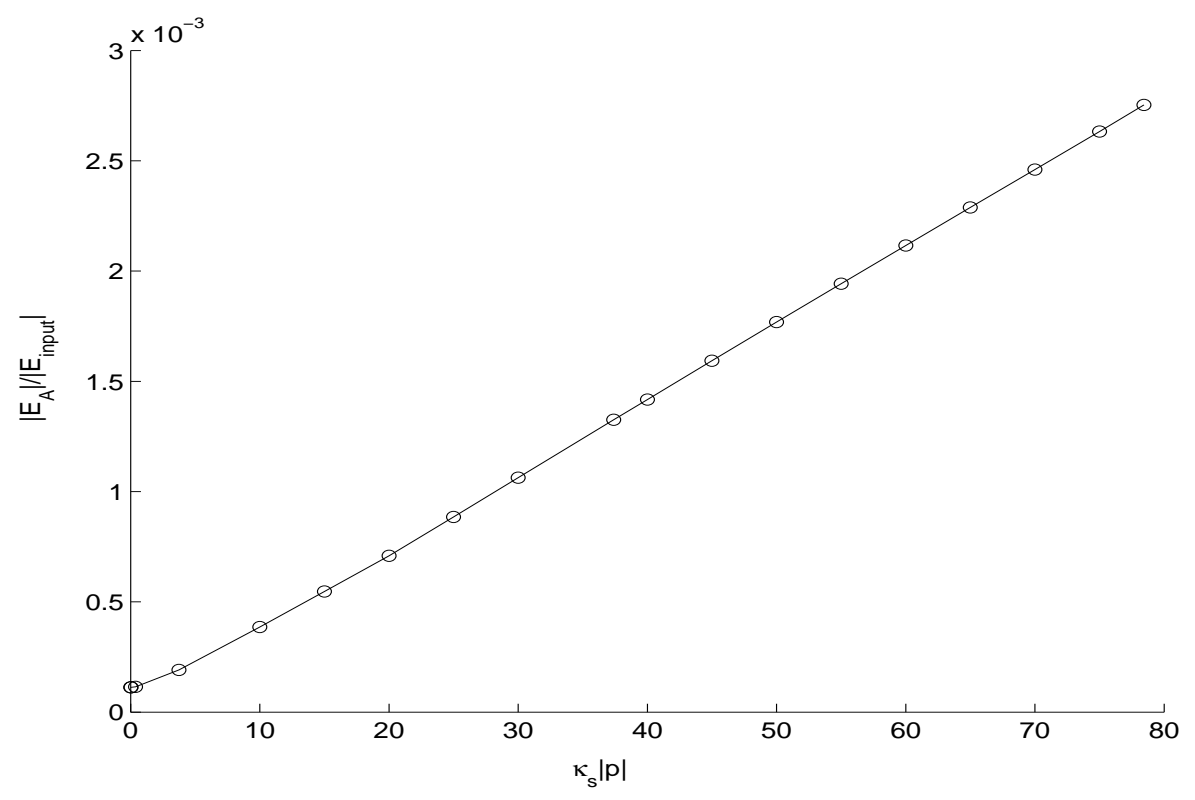

Figure 29: Plot of the ratio $\left|E_{A}\right| /\left|E_{\text {input }}\right|$ versus the product $\kappa_{s}|p|$.

We plot the ratio of the maximum amplitude of the reflected wave to the maximum amplitude of the input wave, since the amplitude of the reflected wave scales with the amplitude of the input wave. We plot these values as a function of the product $\kappa_{s}|p|$, since the strength of the reflection scales with this product. In this way, the curve can be used in a universal manner. Knowing the pressure dependence $\kappa_{s}$ of a material, one can determine the magnitude of the pressure wave necessary to achieve a desired signal-to-input ratio. Similarly, if one knows the inherent noise in making observations of the reflected waves, one can determine the size of the input source needed to achieve a satisfactory signal-to-noise ratio, i.e., to produce a detectable effect.

Figure 30 depicts the same data as Fig. 29, except on a $\log \mathrm{x}$ scale. With this view, it can be seen that the values for the ratio $\left|E_{A}\right| / \mid E_{\text {input }}$ plateau for small values of $\kappa_{s}|p|$. Simulations with different grid refinements show that this plateau tends toward zero as the mesh size decreases.

We can use these curves to estimate the amplitude of the pressure wave that we would need in order to observe a reflection of the EM pulse in our experiments. The magnitude of the incident electric field in the experiment is roughly $15 \mathrm{~V} / \mathrm{m}$. If we look for the amplitude of the reflected pulse to be roughly $15 \mathrm{mV} / \mathrm{m}$ (in order to be above the noise), then we would look for a ratio $\left|E_{A}\right| /\left|E_{\text {input }}\right|=1 \times 10^{-3}$. This would require that $\kappa_{s}|p| \approx 30$. Since $\kappa_{s}=3.74 \times 10^{-8}$ for water (agar), we estimate that we would need a pressure amplitude $|p| \approx 8 \times 10^{8} \mathrm{~Pa}$, or 8000 atmospheres. 


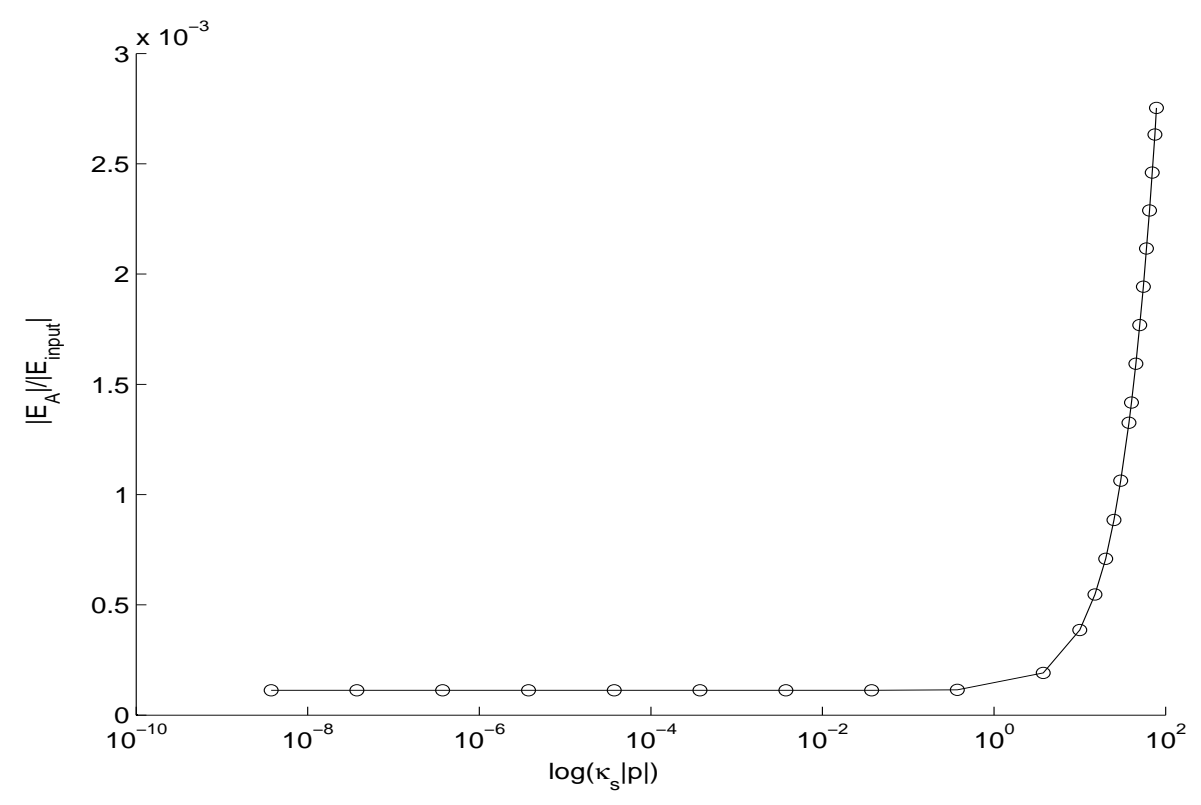

Figure 30: Plot of the ratio $\left|E_{A}\right| /\left|E_{\text {input }}\right|$ versus the product $\log \left(\kappa_{s}|p|\right)$.

\section{CONCLUSION}

In summary, we have constructed a workable experimental antenna where we can launch EM waves at an agar target and make TDR measurements. The circuitry and timing necessary to initiate an acoustic wave and synchronize it with the EM pulse have been built. We have carried out TDR experiments with different delay times, pulse widths, and target thicknesses.

To date, there is no evidence of EM reflection from the acoustic wave front for the range of inputs in which our experimental antenna can be operated. Experimental results are complicated due to jitter in the time base which results in slight offsets and spikes in the subtracted signals. These spikes in the subtracted signal are also observed when the input pulse reflects from the feed of the antenna, further obscuring results in the vicinity of interest. In addition, the long-term oscillations that have been observed in the subtracted data seem not to be the result of acoustic events, but an artifact of the transducer/timer circuitry interacting with the electromagnetic signal in the case when the transducer circuit is connected to the timing circuit.

Evaluation of the expected pressure dependence of the agar indicates that it would be difficult to observe EM reflection from the acoustic wave front with our current transducer, where the magnitude of the pressure wave is estimated to be on the order of $0.1 \mathrm{~Pa}$. range. A suitable source for acoustic waves must satisfy a difficult set of constraints. It must be much more powerful than our current transducer, must be remotely controlled for synchronization of the EM and acoustic signals, must produce a high frequency pulse that produces a sharp wave front in the material, and, ideally, would not interact with the EM field in the antenna (no wires or metal parts).

\section{Acknowledgements}

This research has been suported in part by the US Air Force Office of Scientific Research under grant AFOSR FA 9550-04-1-0220. 


\section{References}

[1] R.A. Albanese, H.T. Banks, J.K. Raye, Nondestructive evaluation of materials using pulsed microwave interrogating signal and acoustic wave induced reflections, Inverse Problems 18 (2002), 1935-1958.

[2] J.C. Anderson, Dielectrics, Chapman and Hall, London, 1967.

[3] H.T. Banks and V. A. Bokil, A computational and statistical framework for multidimensional domain acoustooptic material interrogation, Quarterly of Applied Mathematics 63 (2005), 156-200.

[4] H.T. Banks, M.W. Buksas and T. Lin, Electromagnetic Material Interrogation Using Conductive Interfaces and Acoustic Wavefronts, Frontiers in Applied Mathematics, Vol. FR21, SIAM, Philadelphia, PA, 2000 .

[5] H.T. Banks, N.L. Gibson and W.P. Winfree, Gap detection with electromagnetic terahertz signals, Nonlinear Analysis: Real World Applications 6 (2005), 381-416.

[6] D.J. Bradley and K.S. Pitzer, Dielectric properties of water and Debye-H $u$ ckel parameters to $350{ }^{\circ} \mathrm{C}$ and 1 kar, J. Phys. Chem 83 (1979), 1599-1603.

[7] L. Brillouin, Wave Propagation and Group Velocity, Academic Press, New York, N.Y., 1960.

[8] CRC Handbook of Chemistry and Physics, 3rd electronic ed., Knovel, Norwich, N.Y., 2000.

[9] D.P. Fernandez, A.R.H. Goodwin, E.W. Lemmon, J.M.H. Levelt Sengers, R.C. Williams, A formulation for the static permittivity of water and steam at temperatures form $238 \mathrm{~K}$ to $873 \mathrm{~K}$ at pressures up to $1200 \mathrm{MPa}$, including derivative and Debye-Hu uckel coefficients, J. Phys. Chem. Ref. Data 26 (1997), $1125-1166$.

[10] W.B. Floriano and M.A.C. Nascimento, Dielectric constant and density of water as a function of pressure at constant temperature, Brazilian Journal of Physics 34 (2004), 38-41.

[11] A. Korpel, Acoustics-optics, Marcel-Dekker, New York, 1997.

[12] E.R. Likhachev, Dependence of water viscosity on temperature and pressure, Technical Physics 48 (2003), 514-515.

[13] N. Miura, S. Yahihara, and S. Mashimo, Microwave dielectric properties of solid and liquid foods investigated by time-domain reflectometry, J. Food Science 68 (2003), 1396-1403.

[14] P. Morse and K. Ingard, Theoretical Acoustics, McGraw-Hill, New York, 1968.

[15] B.B. Owen, R.C. Miller, C.E. Milner, and H.L. Cogan, The dielectric constant of water as a function of temperature and pressure, J. Phys. Chem 65 (1961), 2065-2070.

[16] V.V. Shcherbakov, Effect of temperature and pressure on dielectric parameters and the limiting highfrequency conductivity of water, Russian Journal of Electrochemistry 34 (1998),--. 